\title{
KFK-405
}

KERNFORSCHUNGSZENTRUM

KARLSRU HE

Institut für Radiochemie

Die Chemie des Protactiniums

C. Keller

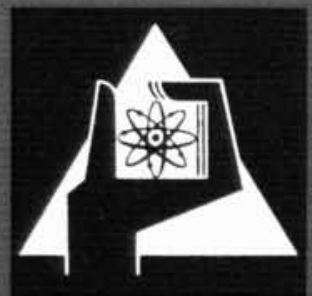





\title{
SONDERDRUCK
}

\section{Die Chemie des Protactiniums}

\author{
VON PRIV.-DOZ. DR. C. KELLER
}

LEHRSTUHL FƯR RADIOCHEMIE, TECHNISCHE HOCHSCHULE KARLSRUHE, UND INSTITUT FÚR RADIOCHEMIE, GESELLSCHAFT FƯR KERNFORSCHUNG MBH., KERNFORSCHUNGSZENTRUM KARISRUHE

Herrn Professor W. Klemm zum 70. Geburtstag gewidmet

\begin{abstract}
Protactinium zeigt in seinen chemischen Reaktionen ein recht zwiespältiges Verhalten. In nichtwäßrigen Lösungsmitteln sowie bei festkörperchemischen Untersuchungen besitzt sowohl vier-als auch fünfwertiges Protactinium Eigenschaften, die es als typisches Actinidenelement charakterisieren. Dagegen erweist sich fünfwertiges Protactinium bei Untersuchungen in wäßriger Lösung als Homologes der Elemente Niob und Tantal. Es zeigt - außer in flußsauren Lösungen - eine starke Tendenz zu irreversiblen Hydrolysen- und Kondensationsreaktionen, was dazu führt, daß sich Tracermengen (ca. 10-12 Molll) und makroskopische Mengen $\left(10^{-3}-10^{-6} \mathrm{Mol} / \mathrm{l}\right)$ Protactinium häufig unterschiedlich verhalten. Durch $R e^{-}$ duktion mit starken Reduktionsmitteln erhält man wäßrige Lösungen von Pa(IV), dessen Eigenschaften wieder weitgehend denjenigen von Th(IV) und $U(I V)$ entsprechen und keine Beziehungen zu $\mathrm{Nb}(\mathrm{IV})$ aufweisen. Unsere Kennthisse vom chemischen Verhalten dieses seltenen Radioelements wurden besonders durch die Gewinnung von $100 \mathrm{~g}$ reinen Protactiniums - der bisher größten Menge - aus Rückständen der englischen Uranfabrikation in den Jahren 1958-1960 gefördert.
\end{abstract}

\section{Einleitung}

Protactinium, das Element mit der Ordnungszahl 91, steht im Periodensystem der Elemente zwischen Thorium und Uran. Aufgrund der radioaktiven Verschiebungssätze sagten Fajans, Russell und Soddy 1913 voraus, daß der Zerfall von UI(238U) in UII(234U) über je ein Isotop des Thoriums und des damals noch unbekannten Ele- ments 91 führen muß. Kurze Zeit später fanden Fajans und Göllring ${ }^{[1]}$ dieses Zwischenglied $\left(\mathrm{UX}_{2}=234 \mathrm{mPa}\right)$ und gaben ihm - wegen der kurzen Halbwertszeit von 1,2 min - den Namen ,Brevium“. 1918 isolierten Hahn und Meitner ${ }^{[2]}$ aus Pechblenderückständen ein langlebiges Isotop des $\mathrm{UX}_{2}$ und nannten es, da es die Mutter-

[1] K. Fajans u. O. Gohring, Naturwissenschaften 1,339 (1913).

[2] O. Hahn u. L. Meimer, Physik. Z. 19, 208 (1918). 
Tabelle 1. Isotope des Protactiniums [5].

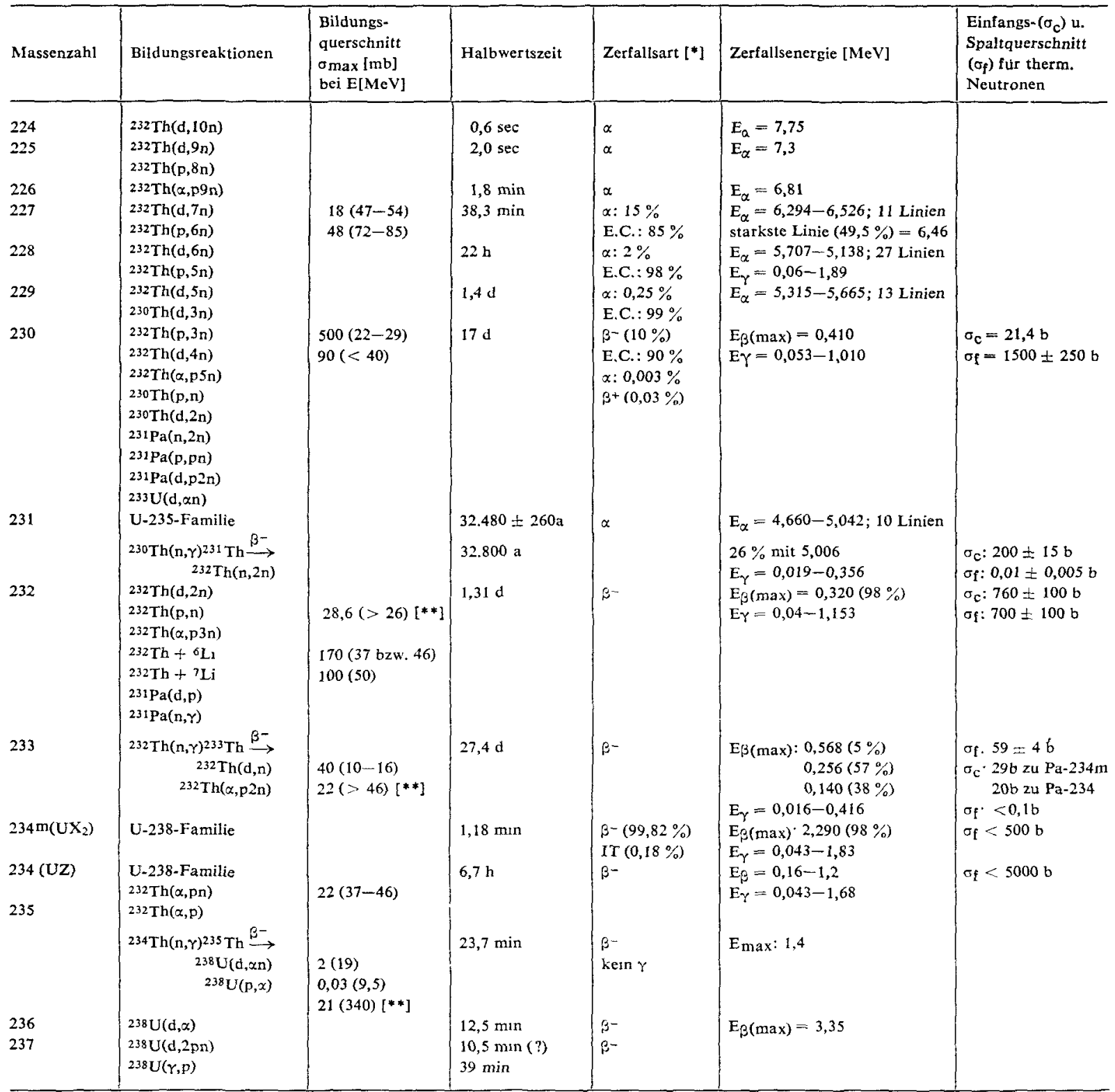

[*] IT = isomerer Ubergang (isomeric transition); E C. = Elektroneneinfang (electron capture).

[**] Nicht $\sigma_{\max }$.

substanz des Actiniums ist, ,Protoactinium“, was später auf „Protactinium“ verkürzt wurde. Dieser Name wurde später international angenommen. Mit der Entdeckung der UZ, des Grundzustandes von ${ }^{234} \mathrm{~Pa}$, fand Hahn das erste Beispiel einer Kernisomerie [3].

Die ersten chemıschen Untersuchungen mit makroskopischen Mengen Protactinium führte $v$. Grosse Ende der zwanziger Jahre im Hahnschen Institut aus, eine eingehendere Bearbeitung begann jedoch erst im 2. Weltkrieg im Rahmen des „Manhattan Project". Zahlreiche Arbeiten über das Protactinium stammen seit den dreißiger Jahren auch aus dem Curie-Laboratorium-Institut du Radium in Paris; so konnten z. B. Haissinsky und Bouissières eine niedrigere Wertigkeitsstufe des Protactiniums, das $\mathrm{Pa}(\mathrm{IV})$, nachweisen [4].

[3] O. Hahn, Naturwissenschaften 9, 84 (1921).

[4] M. Haissinsky u. G. Bouissières, C. R. hebd. Séances Acad. Sci. 226, 573 (1948).

[5] Eine ausgezeichnete und weitgehend vollstandige Literaturübersicht über die Zerfallsdaten der Pa-Isotope findet sich in:

E. K. Hyde, I. Perlman u. G.T. Seaborg: The Nuclear Properties of the Heavy Elements. Prentice Hall, Englewood Cliffs 1964, Bd. II, S. 693.
Aber erst die Isolierung von $100 \mathrm{~g} 231 \mathrm{~Pa}$ in Springfield und Harwell um 1960 erlaubte es, die Chemie dieses seltenen Radioelements auf breiter Basis zu erforschen. Das steigende Interesse, das sowohl in wissenschaftlicher als auch in praktischer Beziehung diesem Element gewidmet wird, äußerte sich nicht zuletzt in zwei Symposien (Gatlinburg, 1963, und Orsay, 1965), die sich allein mit der Cheme des Protactiniums befaßten.

\section{Isotope und Kernchemie des Protactiniums}

Bis heute sind 14 Isotope des Protactiniums mit den Massenzahlen 224-227 bekannt (Tabelle 1). Von ihnen besitzen nur drei größere Bedeutung:

${ }^{231} \mathrm{~Pa}$ ist das einzige $\mathrm{Pa}$-Isotop, das eine für Arbeiten mit wägbaren Mengen ausreichende Halbwertszeit aufweist. Zur Gewinnung dieses Isotops ist man heute noch weitgehend auf die Aufarbeitung von Rückständen der Uranproduktion angewiesen. Später dürfte es auch loh- 
nend werden, das mit schnellen Neutronen über die Reaktion

$232 \mathrm{Th}(\mathrm{n}, 2 \mathrm{n}){ }^{231} \mathrm{Th} \stackrel{B^{-}}{\longrightarrow} 231 \mathrm{~Pa}$

Tabelle 2. Berechnete Zusammensetzung einer Th-Probe (100 g) nach Bestrahlung. $\Phi$ (thermisch) $=5 \times 10^{14} \mathrm{n} / \mathrm{cm}^{2} \cdot \mathrm{sec}, \Phi$ (eptthermisch) $=$ 1/12 $\Phi$ (thermısch), Bestrahlungszeit 28 Tage (integraler NeutronenfluB $\left.\mathrm{nvt}=1,2 \cdot 10^{21} \mathrm{n} / \mathrm{cm}^{2}\right)[10,11]$.

\begin{tabular}{l|r}
\hline $232 \mathrm{Th}:$ & $98,6 \mathrm{~g}$ \\
$231 \mathrm{~Pa}:$ & $1 \mathrm{mg}$ \\
$233 \mathrm{~Pa}:$ & $950 \mathrm{mg}$ \\
$233 \mathrm{U}:$ & $320 \mathrm{mg}$ \\
$234 \mathrm{U}:$ & $65 \mathrm{mg}$ \\
$235 \mathrm{U}:$ & $5 \mathrm{mg}$ \\
Spaltprodukte: & $60 \mathrm{mg}$
\end{tabular}

gebildete $231 \mathrm{~Pa}$ aus den Rückständen der ${ }^{232} \mathrm{Th}-233 \mathrm{U}$ Aufarbeitung zu isolieren (Tabelle 2). Der Schwellenwert der ${ }^{232} \mathrm{Th}(\mathrm{n}, 2 \mathrm{n})$-Reaktion liegt bei $6,35 \mathrm{MeV}^{[6]}$, der ${ }^{232} \mathrm{Th}(\mathrm{n}, 2 \mathrm{n})^{231}$ Th-Wirkungsquerschnitt für Neutronen

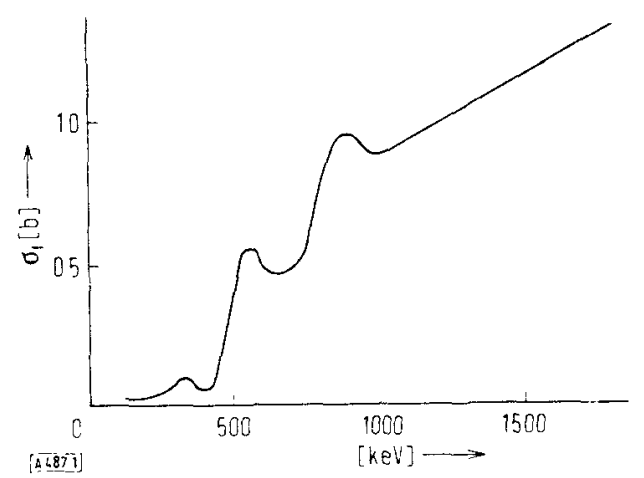

Abb. 1. Abhàngigkeit des ${ }_{91}^{231} \mathrm{~Pa}$-Spaltquerschnitts von der Neutronenenergie.

Ordinate: Spaltquerschnitt $\sigma_{f}[\mathrm{~b}]$.

Abszisse: Energie der Neutronen [keV].

mit der Energieverteilung des Spaltspektrums beträgt $12,4 \pm 0,6 \mathrm{mb}{ }^{[7]}$. Die Spaltschwelle für ${ }^{231} \mathrm{~Pa}$ liegt bei $0,4 \mathrm{MeV}$. Mit steigender Neutronenenergie nimmt der Wirkungsquerschnitt für die Spaltung stark zu (Abb. $1^{[8]}$ ). Die Halbwertszeit der Spontanspaltung von ${ }^{231} \mathrm{~Pa}$ beträgt $1,1 \times 10^{16 \mathrm{a}}{ }^{[9]}$, was 0,3 Spontanspaltungen /min'g entspricht.

${ }^{233} \mathrm{~Pa}$ ist ein $\mathrm{Zwischenprodukt} \mathrm{beim} \mathrm{Briten} \mathrm{von}{ }^{23} \mathrm{U}$ aus 232 Th gemäß

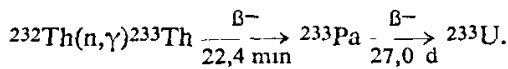

Bei chemischen Untersuchungen wird 233Pa häufig als Markierungsnuklid verwendet.

${ }^{234 \mathrm{~m}} \mathrm{~Pa}$ dient wegen seiner kurzen Halbwertszeit zur Demonstration schneller radiochemischer Trennverfahren [12].

[6] Y. Nishima, T. Yasaki, K. Kimura u. M. Ikawa, Nature (London) 142,874 (1938).

[7] Atomic Energy Research Establishment, Report AERE-R/ R-2366 (1957).

[8] S. M. Dubrovina u. V. A. Shigin, Soviet Phys. Doklady, English Transl. 9, 579 (1963).

[9] E. Segré, Physic. Rev. 86, 21 (1952).

[10] J.W, Codding, J. R. Berreth, R. P. Schuman, W. H. Burgus, R. A. Deal u. F, B. Simpson, Colloque International sur la Physico-Chimie du Protactinium, Orsay/Frankreich, Juli 1965.

[11] J.W. Codding, J. R. Berreth, R. P. Schuman, W. H. Burgus u. R. A. Deal, Report IDO-17007 (1964).

[12] W. Seelmann-Eggebert, C. Keller u. G. Zundel, Kernforschungszentrum Karlsruhe, Bericht KFK-41 (1961).
3. Röntgenspektrum und Elektronenkonfiguration

des Protactiniums

Von Grosse und Mitarbeiter fanden im Röntgenspektrum des ${ }^{231} \mathrm{~Pa} 21$ Linien der L-Serie $\left(\lambda=586,6 \times 10^{-11}\right.$ bis $1088,5 \times 10^{-11} \mathrm{~cm}$ ) und 14 Linien der M-Serie $(.=$ $252,2 \times 10^{-11}$ bis $\left.518,2 \times 10^{-11} \mathrm{~cm}\right)^{[13-15]}$. Zur spektrographischen Bestimmung von ${ }^{231} \mathrm{~Pa}$ sind die Linien bei 3957,8 und 4090,1 $\AA$ am geeignetsten [14].

Nach der ,Actiniden-Hypothese" sollte Protactinium zwei $5 f$-Elektronen besitzen. Aus dem Verlauf der Atomradien ${ }^{[16]}$ sowie dem $\mathrm{Pa}-\mathrm{O}-\mathrm{Abstand}$ im $\mathrm{PaO}{ }^{[17]}$ ist indirekt jedoch auf eine Auffüllung der $6 \mathrm{~d}$-Schale $\mathrm{zu}$ schließen. Dieser Befund stimmt mit Berechnungen von Cap ${ }^{[18]}$ über die Energien der 5f- und 6d-Konfiguration überein. Auch Roof ${ }^{[19]}$ fand bei der Analyse der Röntgen-Absorptionskoeffizienten keine Anzeichen für 5fElektronen, während Couchois ${ }^{[20]}$ aus Unregelmäßigkeiten in den Röntgen-Absorptionskoeffizienten - in Übereinstimmung mit theoretischen Überlegungen von Friedel ${ }^{\{21]}$ - schloß, daß in Th, Pa und U 5f- und 6dElektronen gemischt vorliegen. Dagegen zeigte Jorgensen [22], daß aus dem Absorptionsspektrum von $\mathrm{Pa}(\mathrm{IV})$ auf das Vorhandensein eines 5f-Elektrons zu schließen ist. Aus der Energie der Elektronenniveaus in einem ${ }^{233} \mathrm{~Pa}-S t r a h 1{ }^{[23]}$ sowie aus optischen und magnetischen Untersuchungen über $\mathrm{Pa}(\mathrm{IV})$-dotiertes $\mathrm{Cs}_{2} \mathrm{ZrCl}_{6}$ ist ebenfalls eine $5 f$-Anordnung zu folgern $[24,25]$. Aus den skizzierten Angaben geht hervor, daß noch keine Klarheit über die Besetzung der 5f- oder 6d-Orbitale des $\mathrm{Pa}$ herrscht. Infolge der geringen Energiedifferenzen der beiden Elektronenkonfigurationen ist sicher ein leichter 5f-6d-Übergang möglich.

\section{Herstellung von reinem ${ }^{231} \mathbf{P a}$ und $233 \mathrm{~Pa}$}

4.1. Anreicherung und Reindarstellung von $23 ! \mathrm{Pa}$ aus Abfallprodukten der Uranproduktion

$231 \mathrm{~Pa}$ kommt als sekundäres Zerfalsprodukt von $235 \mathrm{U}$ in Uranmineralien in etwa gleicher Menge wie Radium vor (Gleichgewichtskonzentration $0,3 \mathrm{ppm}$ ). Die Darstellung des ersten reinen $\mathrm{Pa}$-Präparats gelang 1 . Grosse

[13] A.v. Grosse, Physic. Rev. 55, 584 (1939).

[14] F. S.Tomkins u. M. Fred, J. opt. Soc. Amer. 39, 357 (1949). [15] E. W'. Richards u. N. J. Alterton, Atomic Energy Research Establishment, Report AERE/R-3851 (1962)

[16] W. H. Zachariasen, Acta crystallogr. 5, 19 (1952).

[17] S. Fried u. W. H. Zachariasen, Proc. 1st Int. Conf. on Peaceful Uses of Atomic Energy, Genf (1958), Paper 730.

[18] F. Cap, Experienta 6, 291 (1950).

[19] R. B. Roof, Physic. Rev. 113, 820 (1959).

[20] $Y$. Colchois, J. Physique Radium 14, 73 (1963).

[21] J. Frtedel, Proc. physic. Soc. (London) 66 A, 333 (1963).

[22] C. K. Jorgensen, Thesis, Universitat Kopenhagen, 1957.

[23] J.Winocur, University of Calfornia, Lawrence Radiation Laboratory, Report UCRL-9174 (1960).

[24] J. D. Axe, H. J. Stapleton u. R. Kyi, J. chem. Physics 32, 1261 (1960).

[25] J. D. Axe, H. J. Stapleton u. C. D. Jefferies, Physic. Rev. 121,1630 (1960). 
1927 [26]. Aus einem von Hahn und Meitner [27] hergestellten Pa-Konzentrat (6 mg 231 Pa in $525 \mathrm{~g}$ ) isolierte er $2 \mathrm{mg}{ }^{231} \mathrm{~Pa}_{2} \mathrm{O}_{5}$. Unter Verwendung eines nur auf Fällungsprozessen aufgebauten Verfahrens gewannen $v$.

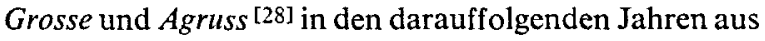
$1000 \mathrm{~kg}$ Rückständen $\left(60 \% \mathrm{SiO}_{2}\right)$ der Joachimsthaler Erzaufbereitung $100 \mathrm{mg}$ reines $231 \mathrm{~Pa}_{2} \mathrm{O}_{5}$. Die Hauptschritte des Trennverfahrens bestanden in der $\mathrm{SiO}_{2}-\mathrm{Ab}$ trennung über eine Alkalihydroxidschmelze und im Auslaugen des gebildeten Silicats mit Wasser. Die ${ }^{231} \mathrm{~Pa}-\mathrm{An}-$ reicherung erfolgte über mehrere $\mathrm{ZrP}_{2} \mathrm{O}_{7}$-Fällungen, während die $\mathbf{Z r}-\mathrm{Pa}$-Trennung durch wiederholte fraktionierte Kristallisationen von $\mathrm{ZrOCl}_{2}$ aus 25-proz. Salzsäure gelang.

Nach 1945 wurden mehrere Verfahren zur Gewinnung wägbarer Mengen $231 \mathrm{~Pa}$ ausgearbeitet [29-35]. Zur ersten Anreicherung der im ppm-Bereich vorliegenden $\mathrm{Pa}-$ Mengen dienten immer Mitfällungsprozesse, während die Grob- und Feinreinigung weitgehend unter Verwendung von Extraktions- oder Ionenaustauschverfahren erfolgte. Nach einem russischen Verfahren ${ }^{[29]}$ werden bei der Ausfällung von $\mathrm{MnO}_{2}$ aus $>1 \mathrm{M} \mathrm{HNO}_{3}$ über $95 \%$ des Pa mitgefällt. Ein französisches Verfahren ${ }^{[30]}$ reduziert $\mathrm{Pa}(\mathrm{V})$ mit $\mathrm{Zn}$-Amalgam zu $\mathrm{Pa}(\mathrm{IV})$ und fällt $\mathrm{PaF}_{4}$. Bei einem Aufbereitungsprozeß der "MoundLaboratories" [31] erfolgt die Anreicherung des Pa über eine Titanphosphatfällung.

In den Jahren 1950-1960 wurden in England aus Abfällen der Uranproduktion in Springfield über $100 \mathrm{~g}$ 231 Pa gewonnen [32-33]. Als Ausgangsmaterial dienten ca. 40 t Rückstände (,,ethereal sludge“) der Urangewinnung mit ca. 3 ppm Pa. Die Rückstände wurden mit kalter $4 \mathrm{~N}$ Salpetersäure (2 Liter $/ \mathrm{kg}$ ) ausgelaugt. Aus der erhaltenen Lösung wurde das Uran mit Tributylphosphat extrahiert und anschließend durch Zugabe von Ammoniak ein Hydroxidniederschlag ausgefällt, welcher zu einer $1 \mathrm{~N}$ salzsauren Lösung aufgelöst wurde. Nach Zugabe von Aluminiumchlorid bildete sich ein Niederschlag, der $75 \%$ des ursprünglichen Pa enthielt. Das Fällungsprodukt wurde nach Digerieren mit warmer 30-proz. Natronlauge (Entfernung von $\mathrm{Al}, \mathrm{Si}, \mathrm{P}$ ) in konzentrierter Salzsäure gelöst. Durch Extraktion mit Diisobutylketon (DIBK) wurden $95 \%$ des Pa der Lö-

[26] A.v. Grosse, Ber. dtsch. chem. Ges. 61, 233 (1928).

[27] O. Hahn u. L. Meitner, Ber. dtsch. chem. Ges. 52, 1812 (1919).

[28] A.v. Grosse u. M. Agruss, J. Amer, chem. Soc. 56, 2200 (1934).

[29] V. S. Spitsyn u. R. A. D'Yachkova, Atomenergle (russ.) 16. 134 (1964); siehe auch Nuclear Energy, Part A and B. 18, 731 (1964).

[30] G. Bouissières u. M. Haissinsky, Bull. Soc. chım. France 18 , 557 (1951).

[31] H.W. Kirby: The Radiochemistry of Protactinum. Natl. Academy of Sciences, Natl. Research Comc1l, Nucl. Science Series NAS-NS-3016, Washington 1959, S. 65.

[32] D. A. Collins, J. J. Hilary, J. S. Nairn u. G. M. Philipps, J, inorg. nuclear Chem. 24, 441 (1962).

[33] N. Jackson, F. J. G. Rogers u. J. Short, Atomic Energy Research Establishment, Report AERE/R-3311 (1960).

[34] M. L. Salutsky, K. Sharer, A. Elmlinger u. M. L. Curtis, J. inorg, nuclear Chem. 3, 289 (1956).

[35] J. S. Nairn, D. A. Collins, H. A. McKay u. A. G. Maddock, Proc. 2nd Int. Conf. on the Peaceful Uses of Atomic Energy, Genf (1957). United Nations Publications, Genf 1958, Bd. 27, S. 16. sung abgetrennt, die Rückextraktion aus der organischen Phase mit $8 \mathrm{~N} \mathrm{HCl}+0,5 \mathrm{~N} \mathrm{HF}$ führte zu 52,4 Liter Lösung mit insgesamt 108,2 g 231 Pa. Die Feinreinigung erfolgte durch Anionenaustausch.

\subsection{Abtrennung von ${ }^{233} \mathrm{~Pa}$ aus bestrahltem Thorium}

Bestrahltes Thoriummetall wird in einer Mischung von konz. $\mathrm{HCl}+0,2 \mathrm{~N} \mathrm{HF}$ gelöst und nach Zugabe von Borax oder $\mathrm{AlCl}_{3}$ - zur Bindung der Fluorid-Ionen die Lösung auf eine kleine Säule $(\mathrm{h}=55 \mathrm{~mm}, \varnothing=3 \mathrm{~mm})$ mit Anionenaustauscher (Dowex-1) gegeben [36]. Pa, $\mathrm{Zr}$ und $\mathrm{Nb}$ bleiben auf der Säule, Th wird nicht adsorbiert. Mit $6 \mathrm{~N} \mathrm{HCl}$ wird $\mathrm{Zr}$ eluiert, anschließend das $\mathrm{Pa}$ mit $9 \mathrm{~N} \mathrm{HCl}+0,1 \mathrm{~N} \mathrm{HF}$. Das Pa-Eluat wird mit dem gleichen Volumen Diisopropylketon (DIPK) geschüttelt, wobei Spuren von $\mathrm{Fe}(\mathrm{III}), \mathrm{Sn}(\mathrm{IV})$ und $\mathrm{Nb}$ (V) extrahiert werden. Nach Zugabe von Borax zur wäßrigen Lösung wird $\mathrm{Pa}(\mathrm{V})$ mit frischen DIPK extrahiert und dabei von $\mathrm{U}, \mathrm{Th}, \mathrm{Ti}, \mathrm{Zr}$ usw. getrennt. Nach Rückextraktion mit $2 \mathrm{~N} \mathrm{HCl}$ wird das $\mathrm{Pa}(\mathrm{V})$ erneut aus $10 \mathrm{~N} \mathrm{HCl}$ auf einer Anionenaustauschersäule $(\mathrm{h}=12 \mathrm{~mm} ; \varnothing=3 \mathrm{~mm})$ adsorbiert und mit 2,7 $\mathrm{N} \mathrm{HCl}$ eluiert. Die ersten 3-6 Tropfen enthalten mehr als $90 \%$ des ursprünglichen $233 \mathrm{~Pa}$.

Andere Verfahren zur Reinigung von $233 \mathrm{~Pa}$ benutzen entweder eine Mitfällung von ${ }^{233} \mathrm{~Pa}$ mit $\mathrm{MnO}_{2}{ }^{[37}$, 38], $\mathrm{PbO}_{2}$ oder $\mathrm{SnO}_{2}{ }^{[39]}, \mathrm{Nb}_{2} \mathrm{O}_{5}{ }^{[40]}, \mathrm{BaZrF}_{6}{ }^{[41]}$, Zirkonphosphat ${ }^{[42]}$, Zirkonmandelat ${ }^{[43]}$ oder Zirkonjodat $[42,44]$ oder Extraktionsmethoden, z.B. die Extraktion mit tertiären Aminen[45], Extraktoon mit Thionyltrifluoraceton (TTA) ${ }^{446,47]}$, Tributylphosphat (TBP) ${ }^{[48]}$ oder Tributylphosphinoxyd [49], oder mit verzweigten Ketonen und Alkoholen [50].

Die Abtrennung des $\mathrm{Pa}$ von Th durch Mitfallung mit $\mathrm{MnO}_{2}$ benutzten Codding et al. [10,11] zur Gewinnung von $1 \mathrm{~g}$ ${ }^{233} \mathrm{~Pa}$ (ca. $2 \times 10^{4} \mathrm{C}$ ) aus $200 \mathrm{~g}$ bestrahltem Th-Metall. Die letzte Reinigungsstufe bestand hier in einer Fällung von PaPerjodat. Die Wärmeentwicklung des reinen Präparats reichte aus, um dieses zu trocknen und das Perjodat partiell zu zersetzen.

[36] M.W. Hill, University of Californa Radiation Laboratory, Report UCRL-8423 (1958).

[37] L. J. Katzin u. R.W. Stoughton, J. inorg. nuclear Chem. 3, 229 (1956).

[38] A. G. Maddock u. G. L. Miles, J. chem. Soc. (London) Suppl. 1949, 255.

[39] C. A. Goodall u. R. L. Moore, J. morg. nuclear Chem. II, 290 (1959).

[40] A. J. Fudge u. J. L. Woodhead, Analyst 81, 417 (1956).

[41] W.W. Meinke, Atomic Energy of Canada, Ltd., Chalk River, Report AECD-2738 (1946).

[42] G.T. Seaborg, J.W. Gofman U. R.W. Stoughton, US.-Pat. 2861866 (1958); Nucl. Sci. Abstr. 12. No. 14396, S. 1724 (1958)

[43] Y. Starik u. L. D. Sheidina, Radıokhmiya $l, 270$ (1959).

[44] J. E Hudgens, B. Warren u. F. L Moore, Report MON$\mathrm{N}-234$ (1947).

[45] F. L. Moore, Analytic. Chem. 28, 997 (1956).

[46] W.W. Meinke, J. chem. Physics 20, 754 (1952).

[47] F. Ichikawa u. S. Uruno, Bull. chem. Soc. Japan 33, 569 (1960).

[48] D. F. Peppard, G.W. Mason u. M.V. Gergel, J. Inorg. nuclear Chem. 3, 370 (1957).

[49] T. Ishimori u. M. Sammour, J. atomic. Energy Soc. Japan 3, 410 (1961).

[50] J. R. Oliver, J. R. Meriwether u. R. H. Rainey, Oak Ridge National Laboratory, Report ORNL-2668 (1959). 


\section{Die Chemie des Protactiniums in wäßrigen Lösungen}

\subsection{Ionenspecies in wäßriger Lösung}

Die Chemie des fünfwertigen Protactiniums in wäßriger Lösung ist ähnlich der des Niobs und Tantals sehr kompliziert, wenn man von flußsauren oder schwefelsauren Lösungen absieht. Es ist selbst in konzentrierter Salzsäure, Salpetersäure oder Perchlorsäure praktisch nicht möglich, Lösungen von $\mathrm{Pa}(\mathrm{V})$ zu erhalten, die über einen längeren Zeitraum stabil sind und nicht allmählich unvorhersagbare Veränderungen erleiden. Diese Instabilität ist sowohl bei Lösungen von Tracermengen ${ }^{233} \mathrm{~Pa}$ als auch bei Lösungen von mMol-Mengen $231 \mathrm{~Pa}$ zu beobachten. Im allgemeinen nimmt die Instabilität mit der Pa-Konzentration zu. Es setzt sich daher immer mehr die Ansicht durch, daß es sich bei den irreversiblen Veränderungen nicht um Hydrolysenreaktionen, sondern um Kondensationsreaktionen handelt [51]:

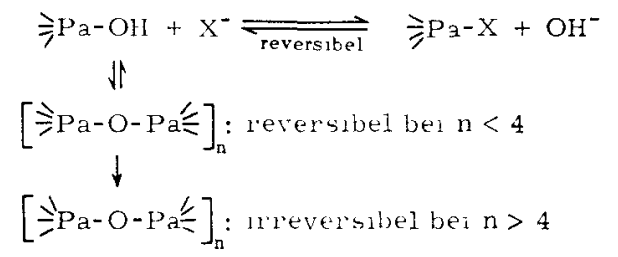

Die Rückbildung der hydroxylhaltigen Species, die allein durch Austauschreaktionen zur Komplexbildung befähigt sind, aus den kondensierten Species verläuft langsam. So erklärt sich z. B. auch, daß das Verteilungsgleichgewicht bei der Extraktion von $\mathrm{Pa}(\mathrm{V})$ mit 0,03 $\mathrm{M}$ Trilaurylamin in Diäthylbenzol ${ }^{[52]}$ oder mit $0,1 \mathrm{M}$ 8Hydroxychinolin in Chloroform [53] auch nach mehreren Tagen noch nicht annähernd erreicht ist.

Nach Extraktionsuntersuchungen am zweiphasigen System $\mathrm{Pa}(\mathrm{V}), \quad(\mathrm{Li}, \mathrm{H}) \mathrm{ClO}_{4} / \mathrm{TTA} \mathrm{C}_{6} \mathrm{H}_{6}$ kam Guillaumont ${ }^{[54-57]} \mathrm{zu}$ dem Schluß, daß bei Tracerkonzentrationen ${ }^{233} \mathrm{~Pa}$ in $1-3 \mathrm{~N} \mathrm{H}^{+}$-Lösungen von $\mathrm{Pa}(\mathrm{V})$ das Ion $\mathrm{Pa}(\mathrm{OH})_{3}^{2+}$ oder $\mathrm{PaO}(\mathrm{OH})^{2+}$ vorliegt, das bei höheren $\mathrm{pH}$-Werten $\mathrm{zu} \mathrm{Pa}(\mathrm{OH})_{2}^{+}$oder $\mathrm{PaO}(\mathrm{OH})_{4}^{+}$hydrolysiert. Aus den Extraktionsdaten ließ sich die Hydrolysenkonstante $\mathrm{K}_{1}$ von $\mathrm{Pa}(\mathrm{OH})_{3}^{2+}$ (Abb. 2) berechnen zu:

$\mathrm{K}_{1}=\left[\mathrm{Pa}(\mathrm{OH})_{4}^{+}\right]\left[\mathrm{H}^{+}\right]\left[\mathrm{Pa}(\mathrm{OH})_{3}^{2+} \mathrm{J}^{-1}=9 \times 10^{-2}\left(\right.\right.$ bei $\left.25^{\circ} \mathrm{C}\right)$.

Demgegenüber sind die Verhältnisse in flußsauren $\mathrm{Pa}(\mathrm{V})$ Lösungen sehr einfach. Aus ramanspektroskopischen Untersuchungen geht hervor, daß die in kristallisierten Doppelfluoriden vorliegenden Komplexionen $\mathrm{PaF}_{6}^{-}$ und $\mathrm{PaF}_{7}^{2-}$ auch in flußsauren Lösungen vorhanden

[51] A. G. Maddock, Colloque International sur la PhysicoChimie du Protactinium, Orsay/Frankreich, Julı 1965.

[52] D. O. Campbell, Colloque International sur la PhysicoChimie du Protactinum, Orsay/Frankreich, Juli 1965.

[53] C. Keller u. K. Mosdzelewski, unveroffentlicht.

[54] R. Guillaumont u. G. Bouissières, Bull. Soc. chim. France 1964, 2098.

[55] R. Guillaumont, Bull. Soc. chim. France 1965, 135.

[56] R. Guillaumont, Colloque International sur la PhysicoChimie du Protactinium, Orsay/Frankreıch, Juli 1965.

[57] R. Guillaumont, C. R. hebd. Séances Acad. Sci, 260, 4739 (1965).
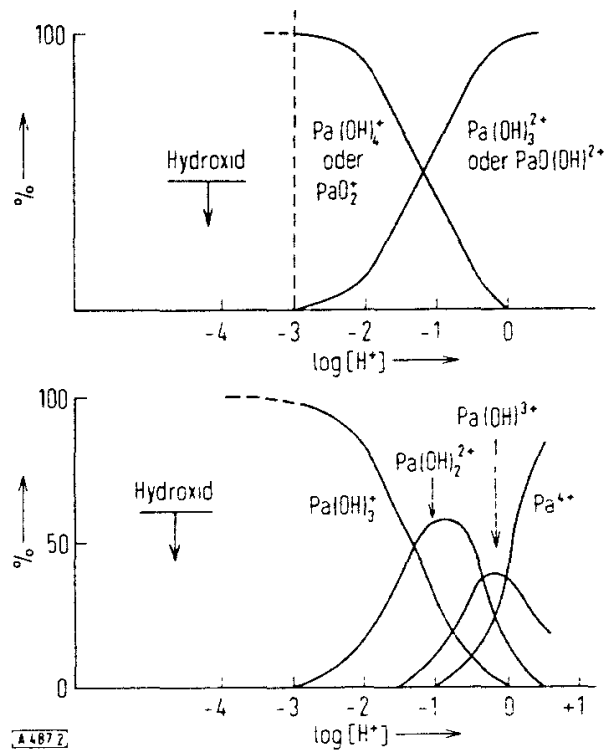

Abb. 2. Hydrolyse von $\mathrm{Pa}(\mathrm{OH})^{2+}$ (oben) und $\mathrm{Pa}^{4+}$ (unten) in Abhang1gkelt von der Saurekonzentratıon (Ionenstärke $\mu=3 \mathrm{M},(\mathrm{L}, \mathrm{H}) \mathrm{ClO}_{4}$ ).

sind [58]. Durch Leitfähigkeitstitration von $\mathrm{Pa}_{2} \mathrm{O}_{5} \cdot \mathrm{aq}$ mit Flußsäure ließen sich alle Zwischenglieder der Reihะ $\mathrm{PaF}^{4+}$ bis $\mathrm{PaF}_{8}^{3-}$ nachweisen.

Dagegen verhält sich $\mathrm{Pa}(\mathrm{IV})$ in mineralsauren Lösungen wie ein echtes Actiniden(IV)-Ion. Die Hydrolyse des $\mathrm{Pa}^{4+}$ erfolgt stufenweise (Abb. 2) über $\mathrm{Pa}(\mathrm{OH})^{3+}(\mathrm{Hy}-$ drolysenkonstante $\left.\mathrm{K}_{1,1}=0,725\right)$ und $\mathrm{Pa}(\mathrm{OH})_{2}^{2}+\left(\mathrm{K}_{1,2}=\right.$ $0,302) \mathrm{zu} \mathrm{Pa}(\mathrm{OH})_{3}^{+}\left(\mathrm{K}_{1,3}=0,017\right)$, bevor $\mathrm{Pa}(\mathrm{OH})_{4}$ ausfallt.

Aus der Änderung des Verteilungskoeffizienten bei Zusatz von Komplexbildnern lassen sich Komplexbildungskonstanten für $\mathrm{Pa}(\mathrm{OH})_{3}^{2+}$ und $\mathrm{Pa}^{4+}$ berechnen (Tab. 3).

Tabelle 3. Komplexbildungskonstanten $\mathrm{K}$ von $\mathrm{Pa}(\mathrm{V})$ und $\mathrm{Pa}(\mathrm{IV})$ (bestimmt aus der Änderung des Vertellungskoeffizienten bel der Extrahtion von Pa mit Thionyltrifluoraceton (TTA) in Gegenwart von Komplesbildnern ( $\mu=3$ M) [55]

\begin{tabular}{l|c|l|l}
\hline \multicolumn{2}{c|}{$\mathrm{Pa}(\mathrm{V})$} & \multicolumn{2}{c}{$\mathrm{Pa}\left(\mathrm{IV}^{\prime}\right)$} \\
\hline Komplex [a] & $\mathrm{K}$ & Komplex & $\mathrm{K}[\mathrm{b}]$ \\
\hline $\mathrm{Pa}(\mathrm{OH})_{3} \mathrm{Cl}^{+}$ & 1,2 & $\mathrm{PaCl}^{3+}$ & 1 \\
$\mathrm{~Pa}(\mathrm{OH})_{2} \mathrm{SO}_{4}^{+}[\mathrm{c}]$ & 20 & $\mathrm{PaCl}_{2}{ }^{2+}$ & 1 \\
$\mathrm{~Pa}(\mathrm{OH})_{2}\left(\mathrm{SO}_{4}\right)_{2}^{-}$ & 320 & $\mathrm{PaSO}_{4}^{++}$ & 42 \\
$\mathrm{~Pa}(\mathrm{OH})_{2}\left(\mathrm{C}_{2} \mathrm{O}_{4}\right)^{+}$ & 150 & $\left.\mathrm{~Pa}^{+} \mathrm{SO}_{4}\right)_{2}$ & $1,53 \cdot 10^{2}$ \\
$\mathrm{~Pa}(\mathrm{OH})_{2}\left(\mathrm{C}_{2} \mathrm{O}_{4}\right)_{2}^{-}$ & $5 \times 10^{4}$ & $\mathrm{PaF}^{3-}$ & $5,35 \times 10^{4}$ \\
$\mathrm{~Pa}(\mathrm{OH})_{2} \mathrm{~F}^{2+}$ & $3,6 \times 10^{3}$ & $\mathrm{PaF}_{2}{ }^{2+}$ & $1,8 \times 10^{8}$ \\
$\mathrm{~Pa}(\mathrm{OH})_{2} \mathrm{~F}_{2}^{+}$ & $4,5 \times 10^{+}$ & & \\
$\mathrm{Pa}(\mathrm{OH})_{2} \mathrm{~F}_{3}$ & $8 \times 10^{10}$ & & \\
& & &
\end{tabular}

[a] Ene Formulserung als Sauerstoffihomplex, z. B. $\mathrm{PaOSO}_{4}{ }^{7}$, ist nicht auszuschließen.

[b] Stets fur dic Reaktion: $\mathrm{Pa}^{4+}+\mathrm{nXm}^{-} \rightleftharpoons \mathrm{PaX}_{\mathrm{n}}(4-\mathrm{n} \mathrm{m})^{+}$

[c] Fur die Reaktion: $\mathrm{Pa}(\mathrm{OH})_{3}{ }^{+}+\mathrm{HSO}_{4}{ }^{-} \rightleftharpoons \mathrm{Pa}(\mathrm{OH})_{2} \mathrm{SO}_{4}{ }^{+}+\mathrm{H}_{2} \mathrm{O}$

Dabei wurde festgestellt, daß die Komplexbildungstendenz von $\mathrm{Pa}^{4+}$ größer als diejenige von $\mathrm{Pa}(\mathrm{OH})_{3}^{2-}$, aber kleiner als die von $\mathrm{Zr}^{4+}$ ist. Die Werte für $\mathrm{Pa}^{4+}$ ordnen sich ausgezeichnet in die Reihe der entsprechenden Actiniden(IV)-Komplexe ein.

[58] O. L. Neller u. A. Chetham-Stiode. Colloque International sur la Physico-Chimı du Protactinium, Orsay/Frankreich, Juli 1965. 


\subsection{Das Extraktionsverhalten von Protactinium}

Über die Extraktion von fünfwertigem Protactinium liegen zahlreiche Arbeiten vor, dagegen sind über $\mathrm{Pa}(\mathrm{IV})$ nur wenige Daten bekannt. Aus den bisherigen Untersuchungen über die Chemie des $\mathrm{Pa}(\mathrm{IV})$ in Lösung ist $\mathrm{zu}$ schließen, daß zwischen Pa(IV) und Th(IV) oder U(IV) keine prinzipiellen, sondern höchstens kleinere graduelle Unterschiede bestehen.

Neben den - als universelle Extraktionsmittel geltenden - Phosphorsäureestern und Aminen sind längerkettige, verzweigte Ketone und Alkohole die besten und spezifischsten Extraktionsmittel für $\mathrm{Pa}(\mathrm{V})$. Dagegen extrahieren Äther und Ester Pa(V) ausgesprochen schlecht. Die höchsten Verteilungskoeffizienten $K_{D}$ sowie die beste Reproduzierbarkeit sind bei der Extraktion aus salzsaurer Lösung gegeben. Geringe Mengen Schwefelsäure setzen - außer bei den Aminen - die Verteilungskoeffizienten stark herab. In salpetersauren oder perchlorsauren Lösungen erfolgt leicht Hydrolyse des $\mathrm{Pa}(\mathrm{V})$ zu polymeren Species, was die schwankenden Ergebnisse verschiedener Autoren erklärt. Die Anwesenheit von Komplexbildnern sowie von $\mathrm{H}_{2} \mathrm{O}_{2}$ verhindert die Extraktion von $\mathrm{Pa}(\mathrm{V})$ weitgehend, bei Gegenwart von Fluoridionen ist eine Extraktion nicht mehr möglich.

\subsubsection{Extraktion mit Phosphorsäureestern}

Bei Verwendung von unverdünntem Tri-n-butylphosphat (TBP) steigt der Verteilungskoeffizient $K_{\mathbf{D}}$ mit der Konzentration an Salzsäure in der wäßrigen Phase [48,59]: in $5 \mathrm{~N} \mathrm{HCl} \mathrm{K}_{\mathrm{D}} \approx 40$, in $8 \mathrm{~N} \mathrm{HCl} \mathrm{K} \mathrm{D}_{\mathrm{D}} \approx 4,5 \times 10^{3}$ und in $12 \mathrm{~N} \mathrm{HCl} \mathrm{K}_{\mathrm{D}} \approx 10^{5}$. Mit Zunahme der Verdünnung des TBP (z.B. durch Benzol) nehmen die Verteilungskoeffizienten $a b[60,61]$. Sie sind in einem weiten Konzentrationsbereich unabhängig von der $\mathrm{Pa}(\mathrm{V})$-Konzentration. Aus der zeitlichen Konstanz von $\mathrm{K}_{\mathrm{D}}$ ist zu schlieBen, daß $10^{-4}-10^{-6} \mathrm{M} \mathrm{Pa}(\mathrm{V})$-Lösungen in ca. $3 \mathrm{~N} \mathrm{HNO}_{3}$ bei $21{ }^{\circ} \mathrm{C}$ mindestens $100 \mathrm{Std}$. stabil sind. Dagegen nimmt $\mathrm{K}_{\mathrm{D}}$ in $1 \mathrm{NHNO}_{3}$ mit der Zeit schnell $\mathrm{ab}$, nach 3 Tagen sind etwa $95 \%$ des $\mathrm{Pa}(\mathrm{V})$ hydrolysiert ${ }^{[62]}$. Bei der Extraktion verschiedener Metallionen mit 20\% TBP/Kerosin aus $2 \mathrm{~N} \mathrm{HNO}_{3}$ nehmen die Verteilungskoeffizienten in folgender Reihe $a b: U(V I)>N p(V l)>$ $\mathrm{Pu}(\mathrm{IV})>\mathrm{Pu}(\mathrm{VI})>\mathrm{Th}(\mathrm{IV})>\mathrm{Np}(\mathrm{IV})>\mathrm{Pa}(\mathrm{V})>$ $\mathrm{Zr}(\mathrm{IV})>\mathrm{Md}(\mathrm{III})$.

Geringe Verunreinigungn an Dibutylphosphat (DBP) im TBP - z.B. durch Hydrolyse oder Radiolyse der $\mathrm{P}-\mathrm{O}-\mathrm{C}$-Bindung gebildet - setzen $\mathrm{K}_{\mathrm{D}}$ stark herauf; so ist z.B. für gereinigtes TBP ( $30 \%$ in Kerosin) aus $6 \mathrm{~N}$ $\mathrm{HNO}_{3} \mathrm{~K}_{\mathrm{D}} \approx 2,6$, für TBP mit $10^{-2} \mathrm{M}$ DBP: $\mathrm{K}_{\mathrm{D}} \approx$ $50^{[60]}$.

Hohe Verteilungskoeffizienten für $\mathrm{Pa}(\mathrm{V})$ weisen auch die Mono- und besonders die Dialkylphosphorsäureester

[59] H. L. Scherff u. G. Hermam, Z. Elektrochem. 64, 1022 (1960).

[60] C. J. Hardy, D. Scargill u. J. M. F/etcher, J. inorg. nuclear Chem. 7, 257 (1958)

[61] S. Shankar, K. S. Venkateswarlu u. C. Copinathan, J. inorg. nuclear Chem. 25, 57 (1963).

[62] C. J. Hardy u. D. Scargill, Proceedngs of the Protactinium Chemistry Symposium Gatinburg/Tenn. (USA), Report TID-7675 (1963), S. 1. (häufig als flüssige Kationenaustauscher bezeichnet) sowie die Phosphonsäureester auf $[60,63-66]$. Im Falle der Extraktion von $\mathrm{Pa}(\mathrm{V})$ mit Di-n-octylphosphorsäureester $(\mathbf{H X})^{[*\}}$ aus salzsaurer Lösung wurde aus dem Verlauf der Verteilungskurve auf die Extraktion von $\mathrm{ClO}\left(\mathrm{HX}_{2}\right) \mathrm{PaX}_{2} \mathrm{~Pa}\left(\mathrm{HX}_{2}\right) \mathrm{OCl}$ geschlossen [67].

\subsubsection{Extraktion mit Aminen}

Amine als Extraktionsmittel - häufig als flüssige Anionenaustauscher bezeichnet - besitzen den Vorteil, daß eine Extraktion auch aus schwefelsaurer Lösung möglich ist. Bei etwa gleicher Zahl der C-Atome des Amins sinkt $\mathrm{K}_{\mathrm{D}}$ in der Reihenfolge primäres Amin $>$ sekundäres Amin $>$ tertiäres Amin. Die Verteilungskoeffizienten sind stark von der Struktur des Amins abhängig. So erhöhen z.B. aromatische Gruppen oder Doppelbindungen in der C-Kette den $K_{D^{-W e r t}}$ beträchtlich [68]. Die Art des organischen Verdünnungsmittels für die Amine ist hierbei nicht ohne Einfluß, z.B. steigt $K_{D}$ für 5-proz. Tributylamin mit dem Logarithmus der Dielektrizitätskonstante des Verdünnungsmittels [69]. Eine strahlenchemische Beeinflussung der Extraktion von $\mathrm{Pa}(\mathrm{V})$ wurde für ein sekundäres Amin bis zu einer Dosis von $5 \times 10^{7} \mathrm{r} 60 \mathrm{Co}-\gamma$-Strahlen nicht festgestellt [70].

Mit Zunahme der Schwefelsaurekonzentration der wäßrigen Phase nehmen die Verteilungskoeffizienten $a b^{[68,69,71]}$. Es wurden zwei verschiedene Steigungen der Extraktionskurven mit einem Knickpunkt bei 7-9 $\mathrm{M} \mathrm{H}_{2} \mathrm{SO}_{4}$ erhalten. Bei der Extraktion mit Tribenzylamin enthält der extrahierte $\mathrm{Pa}(\mathrm{V})$ Sulfat-Komplex im Bereich 0,4-0,9 $\mathrm{M} \mathrm{H}_{2} \mathrm{SO}_{4}$ zwei Moleküle Amin pro Pa-Atom [72].

Nitrobenzol und Benzonitril als Extraktionsmittel weisen im Vergleich zu den Ketonen keine Vorteile auf, eine mehr als 95-proz. Extraktion von $\mathrm{Pa}(\mathrm{V})$ erfolgt erst in 7,5 bzw. 8,5 N $\mathrm{HCl}[69]$.

Wahrend $\mathrm{Pa}(\mathrm{V})$ schon aus etwa $6 \mathrm{~N} \mathrm{HCl}$ mit 5-proz. Tri-noctylamin quantitativ extrahiert wird, wird $\mathrm{Pa}(\mathrm{IV})$ erst aus $10 \mathrm{~N} \mathrm{HCl}$ zu mehr als $95 \%$ in die organische Phase übergeführt [73].

[63] V. B. Sherchenko, V. A. Mikhailov u. Y. P. Zavalskii, J. anorg. Chem. (russ.) 3, 1955 (1958).

[64] V. A. Mikhailov, V. B. Sherchenko u. V. A. Kolganov, J. anorg. Chem. (russ.) 3, 1959 (1958).

[65] J. M. Chilton, Proceedings of the Protactinium Chemistry Symposium Gatlinburg/Tenn. (USA), Report TID-7675 (1963), S. 15.

[66] F. E. Butler. Analytic. Chem. 37, 340 (1965).

[*] In Benzollosung als Dimeres $\left(\mathrm{H}_{2} \mathrm{X}_{2}\right)$ vorliegend.

[67] G.W. Mason, C. Andrejasich, S. Lewey u. D. F. Peppard, Colloque international sur la Physico-Chimie du Protactinium, Orsay, Julı 1965.

[68] D. O. Campbell, Proceedings of the Protactunium Chemistry Symposium Gatlinburg/Tenn. (USA), Report TID-7675 (1963), S. 87.

[69] A. G. Gobble u. A. G. Maddoch, J. inorg. nuclear Chem. 7, 94 (1958).

[70] F. Ichikawa u. S. Uruno, Bull. chem. Soc. Japan 33, 569 (1960).

[71] D. Brown, T. Sato, A. J. Smith u. R. G. Wilkins, J. inorg. nuclear Chem. 23, 91 (1961).

[72] R. Muxart, R.Gullaumont u. H.Arapakz-Stapelias, Colloque Internatıonal sur ta Physico-Chimie du Protactınıum, Orsay! Frankreich, Juli 1965.

[73] G. Bouissières, C. Ferraduni, M. Garcon, R. Guillaumont, M. Hussonois, R. Muxart, H. Pezerat, D. Robertson u. T. Stchouzkoy, Proceedings of the Protactinium Chemistry Symposium Gatlinburg/Tenn. (USA), Report TID-7675 (1963), S. 160. 


\subsubsection{Extraktion mit Alkoholen und Ketonen}

Die Verteilungskoeffizienten für $\mathrm{Pa}(\mathrm{V})$ steigen mit der Säurekonzentration der wäßrigen Phase, sie sind bei gleicher Säurekonzentration aus salzsaurer Lösung größer als aus salpetersaurer Lösung. Bei konstanter $\mathrm{HCl}$-Konzentration nimmt $\mathrm{K}_{\mathrm{D}}$ mit der $\mathrm{Cl}^{-}$-Ionenkonzentration zu. Die Extraktion aus $\mathrm{HBr}$-Lösung erfolgt erst bei höheren Säurekonzentrationen als aus $\mathrm{HCl}$-Lösung. Die Solvatationszahl der extrahierten $\mathrm{Pa}(\mathrm{V})$-Species beträgt für Diisobutylketon und Diisopropylketon drei [74], für Amylacetat und TBP [60] dagegen nur zwei. Unter sonst gleichen Bedingungen fällt $\mathrm{K}_{\mathrm{D}}$ in der Reihe Mesityloxid $>$ Diäthylketon $>$ Acetophenon $>$ Methyl-n-hexylketon $>$ Diisobutylketon $>$ Diisopropylketon (Ab-

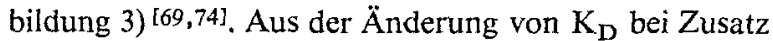
von Fluorid oder Oxalat wurden folgende Reihen der Komplexbildungstendenz festgestellt $[69,75]$ :

gegenuber Fluorid: $\mathrm{Pa}, \mathrm{Z}_{\mathrm{r}}>\mathrm{Nb}>\mathrm{Ta}$

gegenüber Oxalat: $\mathrm{Nb}>\mathrm{Ta}>\mathrm{Pa}, \mathrm{Zr}$

Längerkettige Alkohole wie Diisopropylmethanol $[76,77]$ und Diisobutylmethanol $[59,76]$ besitzen ebenfalls gute Extraktionseigenschaften für $\mathrm{Pa}(\mathrm{V})$. Für Diisobutylcarbinol wurde im Bereich $10^{-4}-10^{-14} \mathrm{M} \mathrm{Pa(V)}$ keine Abhängigkeit des Verteilungskoeffizienten von der $\mathrm{Pa}(\mathrm{V})$-Konzentration festgestellt [78].

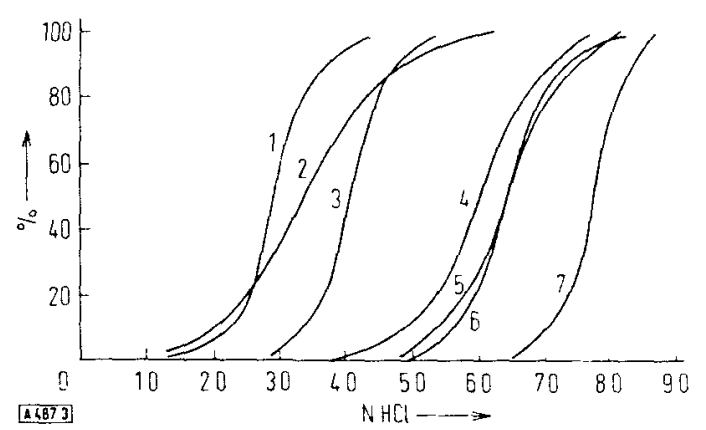

Abb. 3. Extraktion von Spurenmengen Protactinium aus salzsaurer Lösung.

1. Diisopropylcarbinol; 2. Tributylphosphat, 3. Acetophenon;

4. $5 \%$ Trilaurylamin in Benzol; 5. $\beta, \beta^{\prime}$-Dichlordiathylather

6. Benzonitril; 7. Nitroberzol.

Ordinate: Extrahierte Menge [\%].

Abszisse: Normalitat der Salzsaure.

\subsubsection{Extraktion als Chelatkomplex}

Die Chelatkomplexe des Pa(V) mit Derivaten des Hydroxylamins [wie Cupferron (Ammoniumsalz des $\mathrm{N}$ Nitrosophenylhydroxylamins), N-Phenylbenzhydroxamsäure, N-Benzoylphenylhydroxylamin] lassen sich inner-

[74] A. G. Goble, J. Golden u. A. G. Maddock, Canad. J. Chem. 34, 284 (1956).

[75] R. A. Dyachkova u. V. I. Spttsyn, Ukrain. physik. J. (russ.) $31,158(1965)$.

[76] R. E. Elson, G.W. Mason, D. F. Peppard, P. A. Sellers u. M. H. Studier, J. Amer. chem. Soc. 73, 4974 (1951).

[77] J. Golden u. A. G. Maddock, J. inorg. nuclear Chem. 2, 46 (1956).

[78] H. L. Scherff u. G. Hermam, Proceedings of the Protactinium Chemistriy Symposium Gat limburg/Tenn. (USA), Report TID-7675 (1963), S. 105. halb weiter Versuchsbedingungen in verschiedene organische Lösungsmittel extrahieren [38,79-82]. Tartrat und Citrat stören die Extraktion nicht, Oxalat nur geringfügig, während Fluoridionen die Extraktion verhindern. Eine Extraktion mit hoher Ausbeute erfolgt auch mit Salicylsäure bei $\mathrm{pH}=4$ aus $\mathrm{CaCl}_{2}$-gesättigter Lósung

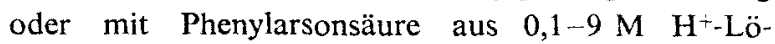
sung $[83,84]$. In Gegenwart von 8-Hydroxychinolin läßt sich $\mathrm{Pa}(\mathrm{V})$ bei $\mathrm{pH}=3-9$ zu über $99,9 \%$ als Chelatkomplex durch Chloroform extrahieren (Abb. 4) [53]. Die Abnahme von $\mathrm{K}_{\mathrm{D}}$ bei $\mathrm{pH}>9$ zeigt, daß das extrahierte Chelat eine zweibasige Säure ist.

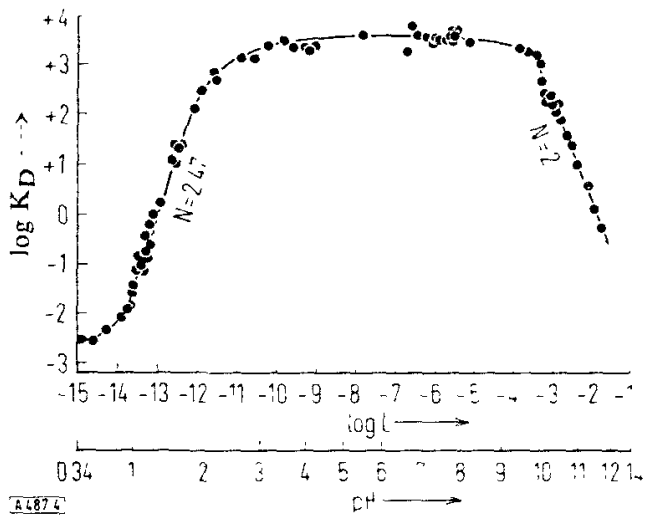

Abb. 4. Extraktion von Spurenmengen $\mathrm{Pa}(\mathrm{V}) \mathrm{mit} 0,1$ M 8-Hydroxychinolin in Chloroform, $\left(u=0,1 \mathrm{M},(\mathrm{Na} . \mathrm{H}) \mathrm{ClO}_{4}\right.$, die Vertellungskoeffizienten $\mathrm{KD}$ w urden durch Ruckextraktion bestımmt)

Abszisse oben. Konzentration L des 8-Hydroxychinolin-Anions. unten: pH-Wert

Die $N$-Werte an der Kurve bedeuten die Kurvensteigung, $d$ h die Zahl der 8-Hydroxychinolin-Mfolekule pro $\mathrm{Pa}$-Atom im extrahierten Komplex.

Eingehend untersucht wurde die Extraktion von $\mathrm{Pa}(\mathrm{V})$ mit Thionyltrifluoraceton (TTA bzw. HX). Eine $0,5 \mathrm{M}$ TTA-Lösung in Benzol extrahiert aus 1-10 N HCl $80-90 \%$ des $\mathrm{Pa}(\mathrm{V})^{[85]}$. Aus der Abnahme ron $\mathrm{K}_{\mathrm{D}}$ in der Reihe $\mathrm{ClO}_{4}^{-}>\mathrm{NO}_{3}^{-}>\mathrm{Cl}^{-}>\mathrm{SO}_{4}^{2-} \cdot \mathrm{F}^{-}$läßt sich der Schluß zichen, daß die Komplexbildungstendenz von $\mathrm{Pa}(\mathrm{V})$ in dieser Reihe zunimmt. Im Bereich 0,5-3 N $\mathrm{HCl}$ werden pro $\mathrm{Pa}(\mathrm{V})$-Atom vier Molekule TTA, im Bereich 0,5-0,015 $\mathrm{N} \mathrm{HCl}$ drei Moleküle gebunden. Daraus wird auf die Extraktion der Species $\mathrm{Pa}(\mathrm{OH})_{3} \mathrm{X}_{2}$. $2 \mathrm{HX}$ bzw. $\mathrm{Pa}(\mathrm{OH})_{4} \mathrm{X} \cdot 2 \mathrm{HX}$ geschlossen $[55,86,87]$. Bei der Extraktion von $\mathrm{Pa}(\mathrm{V})$ aus $3 \mathrm{~N} \mathrm{HClO}_{4}$-Lösung mit einer benzolischen Lösung von TBP + TTA $(1: 1)$ wurde ein synergistischer Effekt festgestellt. Hier wird die Species

[79] J. R. Oliver, J. R. Mermether u. R. H Ratiel, Oak Ridge National Laboratory. Report ORNL-2668 (1959)

[80] B. F. Miassoedov, E. S. Palchine U. P. Y. Palel. J. anorg. Chem. (russ.) 19,105 (1964).

[81] A. V. Lapickis, N. P. Rudenho u. Abdel Garad Saed. Colloque international sur la Physico-Chimie du Protactinium Orsay: Frankreich, Julı 1965.

[82] S.Y.Lyle u. A. D. Shendrikar. Talanta 12.573 (1965)

[83] A. V. Nikolaev, A. G. Kurnakova u. Z G. Rumbantseva. Russ. J. unorg. Chem. 4, 758 (1959).

[84] B. Mjassoedov, E. Palchine 11. P. Palar. Colloque international sur la Physico-Chimie du Protactmintu. Orsay Frankrelch, Jull 1965.

[85] B. Massoedov u. R. Maxart, Bull. Soc. chm. France 1902 237.

[86] F. Boulanger u. R. Guillammont. Bull. Soc. chim. Frunce $1964,3032$.

[87] R. Guillaumont, Bull. Soc. chim. France 1965, 132. 
$\mathrm{Pa}(\mathrm{OH})_{3} \mathrm{X}_{2} \cdot 2$ TBP extrahiert ${ }^{[103]}$. Bei TTA :TBP $\approx 10: 1$ wird dagegen $\mathrm{Pa}(\mathrm{OH})_{3} \mathrm{X}_{2} \cdot \mathrm{HX} \cdot \mathrm{TBP}$ extrahiert. $\mathrm{Pa}(\mathrm{IV})$ wird mit $>0,3 \mathrm{M}$ TTA/Benzol zu etwa $90 \%$ als $\mathrm{PaX}_{4}$ extrahiert ${ }^{[88]}$.

\subsection{Das Ionenaustauschverhalten von Protactinium}

\subsubsection{Anionenaustausch}

Die Adsorption von $\mathrm{Pa}(\mathrm{V})$ aus salpetersaurer und salzsaurer Lösung an Anionenaustauschern nimmt oberhalb $3 \mathrm{M} \mathrm{H}^{+}$mit steigender Säurekonzentration zu [89-93]. Nowikow und Pfrepper [93] fanden im Bereich 1-4 N $\mathrm{HNO}_{3} \mathrm{~Pa}(\mathrm{~V})-\mathrm{NO}_{3}^{-}$-Komplexe der Zusammensetzung $1: 1$ (formuliert als $\left.\left[\mathrm{PaO}_{\mathrm{x}}(\mathrm{OH})_{\mathrm{y}} \mathrm{NO}_{3}\right]\right), \quad 1: 2$ $\left(\left[\mathrm{PaO}_{\mathrm{x}}(\mathrm{OH})_{\mathrm{y}}\left(\mathrm{NO}_{3}\right)_{2}\right]^{-}\right)$und $1: 4\left(\left[\mathrm{PaO}_{\mathrm{x}}(\mathrm{OH})_{\mathrm{y}}\left(\mathrm{NO}_{3}\right)_{4}\right]^{-}\right)$ mit dem Stabilitätskonstanten $\beta_{1}=0,68, \beta_{2}=3,0$ und $\beta_{4}=11,93$ für $1 \mathrm{~N} \mathrm{H}^{+}$. Im Gegensatz zu Angaben von Hardy et al. ${ }^{[60]}$ sollen diese Komplexe vorwiegend anionischer Art sein. Oberhalb $2 \mathrm{~N} \mathrm{H}^{+}$wird $\mathrm{Pa}(\mathrm{V})$ ebenfalls aus $\mathrm{HBr}$ - und HJ-Lösung adsorbiert [92,94], allerdings sind die Verteilungskoeffizienten um etwa zwei Größen-

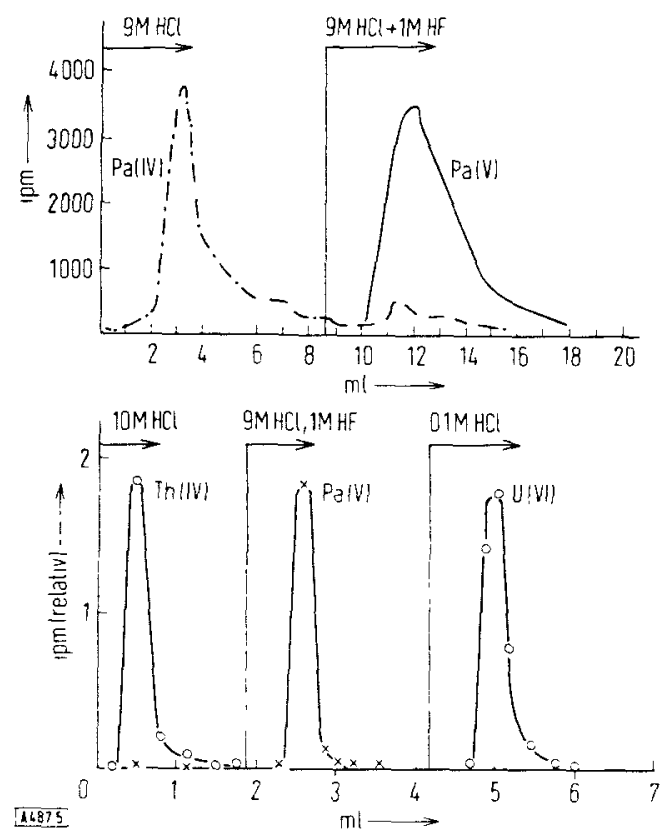

Abb. 5. Ionenaustauschtrennungen mit $\mathrm{Pa}$.

Oben. Trennung von $\mathrm{Pa}(\mathrm{IV})$ und $\mathrm{Pa}(\mathrm{V})$ am Anonenaustauscher Dowex 1

Unten. Trennung von Th(IV), $\mathrm{Pa}(\mathrm{V})$ und $\mathrm{U}(\mathrm{VI})$ am Anionenaustauscher Dowex 1.

Ordinaten: Radıoaktivitat [Impulse/min]

Abszissen: Eluatvolumen [m]

[88] G. Bouissières u. J.Vernots, C. R. hebd. Séances Acad. Sci. 244, 2508 (1957)

[89] S. Kahn u. D. E. Hawkinson, J. inorg. nuclear Chem. 3, 155 (1956)

[90] K. A. Kraus u. G. E. Moore, J. Amer. chem. Soc. 72, 4293 (1950).

[91] L. R. Bunney, N. E. Ballou, J. Pascual u. S. Fot, Analytic. Chem. 31, 324 (1959).

[92] C. Keller, Radiochim. Acta 1,147 (1963).

[93] J. Nowikow u. G. Pfrepper, Z. Naturforsch. 18b, 993 (1963). [94] T. Anderson u. A. B. Knutzen, Acta chem. scand. 16, 849 (1962). ordnungen kleiner als bei der Adsorption aus Salzsäure, was auf eine geringere Tendenz von $\mathrm{Pa}(\mathrm{V})$ zur Komplexbildung mit $\mathrm{Br}^{-}$und $\mathrm{J}^{-}$schließen läßt.

Geringe Mengen Fluoridionen setzen die Adsorption von $\mathrm{Pa}(\mathrm{V})$ aus salzsaurer Lösung an Anionenaustauschern stark herab [95-98], was häufig zur Abtrennung des $\mathrm{Pa}$ benutzt wird (z. B. zu den Trennungen Nb-Ta-Pa oder $\mathrm{Pa}(\mathrm{IV})-\mathrm{Pa}(\mathrm{V})$, Abb. 5). Die Verteilungskoeffizienten in verdünnter reiner Flußsäure sind sehr hoch (ca. $2 \times 10^{4}$ für $0,2 \mathrm{~N} \mathrm{HF}$ ), nehmen jedoch mit steigender HF-Konzentration stark ab (ca. $10^{2}$ in $7 \mathrm{~N} \mathrm{HF}$ ) ${ }^{92,99]}$. Eine ähnliche Abnahme von $K_{D}$ mit der Säurekonzentration ist bei der Adsorption aus schwefelsaurer Lösung festzustellen [71,90-92]. Aus der Abhängigkeit $\mathrm{K}_{\mathrm{D}}=$ $\mathrm{f}\left[\mathrm{H}_{2} \mathrm{SO}_{4}\right]$ folgt, daß zwei $\mathrm{Pa}(\mathrm{V})-\mathrm{SO}_{4}^{2-}$-Komplexe mit den Ladungen -1 bzw. -3 und den Zusammensetzungen $\left[\mathrm{PaO}\left(\mathrm{SO}_{4}\right)_{2}\right]^{-}$und $\left[\mathrm{PaO}\left(\mathrm{SO}_{4}\right)_{3}\right]^{3-}$ existieren. Die Darstellung von $\mathrm{H}_{3} \mathrm{PaO}\left(\mathrm{SO}_{4}\right)_{3}$ (stabil bis ca. $350^{\circ} \mathrm{C}$, hexagonale Struktur mit $\mathrm{a}=9,743 \AA$ und $\mathrm{c}=5,506 \AA$ bei $\mathrm{Z}=2$ ) durch Eindampfen einer ( $\mathrm{HF}+\mathrm{H}_{2} \mathrm{SO}_{4}$ )-haltigen $\mathrm{Pa}(\mathrm{V})$ Lösung sowie von amorphem $\mathrm{HPaO}\left(\mathrm{SO}_{4}\right)_{2}$ und $\mathrm{HPaO}_{2} \mathrm{SO}_{4}$ durch thermische Zersetzung von $\mathrm{H}_{3} \mathrm{PaO}\left(\mathrm{SO}_{4}\right)_{3}$ bestätigen die Annahmen über die Species $\mathrm{Pa}(\mathrm{V})-\mathrm{SO}_{4}^{2-}[100]$. Das Kalium-Salz der Oxotrisu]fatoprotactinium(V)-säure, $\mathrm{K}_{3} \mathrm{PaO}\left(\mathrm{SO}_{4}\right)_{3}$, wurde schon früher dargestellt ${ }^{[101]}$. Auch die entsprechende SelenatoSäure $\mathrm{H}_{3} \mathrm{PaO}\left(\mathrm{SeO}_{4}\right)_{3}$ (hexagonal mit $\mathrm{a}=9,743 \AA$ und $\mathrm{c}=5,679 \AA$ ) konnte isoliert werden [118]. Es sei in diesem Zusammenhang bemerkt, daß von $\mathrm{Nb}(\mathrm{V})$ und $\mathrm{Ta}(\mathrm{V})$ keine analogen Doppelsulfate existieren.

Nach Nowikoff und Pfrepper [93] liegt in 1-2 $\mathrm{M} \mathrm{H}_{2} \mathrm{SO}_{4}$ neben dem 1 : 2-Komplex $\mathrm{Pa}(\mathrm{V}): \mathrm{SO}_{4}^{-}\left(\beta_{2}=7,39\right.$ bei $\left.1,0 \mathrm{~N} \mathrm{H}^{+}\right)$noch ein geringer Anteil eines 1:1-Komplexes $\left(\left[\mathrm{PaO}_{\mathrm{x}}(\mathrm{OH})_{\mathrm{y}} \mathrm{SO}_{4}\right]^{-}\right.$ mit $2 x+y=4 ; \beta_{1}=0,94$ bei $1,0 \mathrm{~N} \mathrm{H}^{+}$) vor.

$\mathrm{Pa}(\mathrm{IV})$ wird aus salzsauren Lösungen nicht an Anionenaustauschern adsorbiert [102]. Auch in schwefel- und flußsauren Losungen besitzt $\mathrm{Pa}(\mathrm{IV})$ nur kleine $\mathrm{K}_{\mathrm{D}}$-Werte ${ }^{[92]}$, daher gleicht es in seinem Verhalten mehr dem Th(IV) als dem U(IV).

\subsubsection{Kationenaustausch}

Die Abtrennung des kurzlebigen $234 \mathrm{mPa}$ von seiner an einen Kationenaustauscher adsorbierten - Muttersubstanz ${ }^{234} \mathrm{Th}$ ist mit einer 5-proz. Citratlösung $(\mathrm{pH}=$ 2,5) möglich [12]. Mit Hilfe von Ionenaustauschverfahren bestimmten Moskvin et al. ${ }^{[103]}$ die Stabilitätskonstanten

[95] F. Nelson, R. M. Rush u. K A. Kraus, J. Amer. chem. Soc. $82,339(1960)$.

[96] K. A. Kraus u. G. E. Moore. J. Amer. chem. Soc. 73, 2900 (1951)

[97] K. A. Kraus, G. E. Moore u. F. Nelson, J. Amer. chem. Soc. 78,2692 (1956)

[98] K. A. Kraus u. G. E. Moore, J. Amer. chem. Soc. 77, 1383 (1955).

[99] A. Chetham-Strode u. O. L. Keller, Colloque international sur la Physico-Chımie du Protactinum, Orsay/Frankreich, Juli 1965.

[100] K.W. Bagnall, D. Brown u. P. J. Jones, J. chem. Soc. (London) $1965,176$.

[101] M. Hatssinsky u. G, Bouissières, Bull. Soc. chım. Francc $1957,557$.

[102] E. Pluchet u. R. Muxart, Bull. Soc. chim. France 1961, 372.

[103] A. J. Moskvm, J. Geleceamu u. A.V. Lapickij, Ber. Akad. Wiss. UdSSR 149, 611 (1963). 
Tabelle 4. Stabilitätskonstanten von Komplexen des $\mathbf{P a}(\mathrm{V})$ mit einigen organischen Sauren $(\mu=0,25 \mathrm{M})$, berechnet aus Jonenaustauschdaten. Eine genaue Formulierung der einzelnen Komplexe ist nicht möglıch, es kann nur das Verhältnıs $\mathbf{P a}(V)$ : Komplexbildner $\mathbf{A}$ angegeben werden [103].

\begin{tabular}{l|l|l}
\hline Saure & $\begin{array}{l}\text { Zusammensetzung } \\
\operatorname{Pa}(\mathrm{V}): A\end{array}$ & $\mathrm{~K}$ \\
\hline Milchsáure & $1: 1$ & $1,7 \times 10^{2}$ \\
\hline $\begin{array}{l}\alpha \text {-Hydroxyiso- } \\
\text { buttersaure }\end{array}$ & $1: 2$ & $3 \times 10^{3}$ \\
\hline Mandelsaure & $1: 3$ & $1,0 \times 10^{7}$ \\
\hline Âpfelsäure & $1: 1$ & $1,0 \times 10^{3}$ \\
\hline Weinsaure & $1: 2$ & $8,3 \times 10^{2}$ \\
& $1: 1$ & $5,3 \times 10^{4}$ \\
\hline Trihydroxyglutarsáure & $1: 2$ & $2.2 \times 10^{2}$ \\
& $1: 1$ & $2,1 \times 10^{4}$ \\
\hline Oxalsaure & $1: 2$ & $9,1 \times 10^{2}$ \\
& $1: 1$ & $7,7 \times 10^{7}$ \\
\hline Citronensaure & $1: 2$ & $3,6 \times 10^{2}$ \\
& $1: 1$ & $8,3 \times 10^{5}$ \\
\hline Aconitsaure & $1: 2$ & $4,5 \times 10^{3}$ \\
\hline Athylendiamin- & $1: 3$ & $8,3 \times 10^{5}$ \\
tetraessigsáure & $1: 1$ & $6,3 \times 10^{8}$ \\
\hline & $1: 1$ & $1,5 \times 10^{2}$ \\
\hline & $1: 2$ & $1,5 \times 10^{7}$ \\
& & $9,1 \times 10^{11}$ \\
\hline
\end{tabular}

zahlreicher Chelate des $\mathrm{Pa}(\mathrm{V})$ mit organischen Säuren. Beim Vergleich der Stabilitätskonstanten von $\mathrm{Pa}(\mathrm{V}) \mathrm{mit}$ anorganischen und organischen Ionen (Tabellen 3 und 4) zeigt sich, daß die meisten organischen Komplexbildner $\mathrm{Pa}(\mathrm{V})$ in ähnlichem Maße stabilisieren wie Schwefelsäure, während Äthylendiamintetraessigsäure $\mathrm{Pa}(\mathrm{V})$ Ionen ebenso stark komplex bindet wie Flußsäure. Dagegen sind Chlorid- und Nitrationen nur äußerst schwache Komplexbildner für $\mathrm{Pa}(\mathrm{V})$. Die Stabilitätskonstanten nehmen mit steigendem Verhältnis Komplexbildner: $\mathrm{Pa}(\mathrm{V})$ im Komplex zu, d.h, die Zahl der freien $\mathrm{Pa}(\mathrm{V})$ Ionen in der Lösung nimmt ab. Dies ist im Prinzip die quantitative Aussage für den allgemein bekannten, qualitativen Befund, daß z.B. die Hydrolysenempfindlichkeit oder die Extrahierbarkeit eines Metallions unter sonst gleichen Bedingungen mit steigendem Komplexbildnerzusatz immer geringer wird.

\subsection{Papierchromatographie und Ionophorese}

Eine papierchromatographische Trennung des $\mathrm{Pa}(\mathrm{V})$ von anderen Elementen ist praktisch nur mit Laufmitteln möglich, die HF enthalten. So erreichte Vernois ${ }^{[104]}$ mit einem Gemisch $\mathrm{HCl} / \mathrm{HF} /$ Butanol eine einwandfreie Trennung $\mathrm{Nb}-\mathrm{Ta}-\mathrm{Pa}$. In flußsäurefreien Laufmitteln erhält man infolge Hydrolyse des $\mathrm{Pa}(\mathrm{V})$ keine guten Trennungen $[105,106]$. Aus der Wanderung von $\mathrm{Pa}(\mathrm{V})$ in alkalischen Laufmitteln schlossen Jakovac und Lederer [107] auf die Existenz eines Protactinat-Anions. Einwandfreie Trennungen sind auch mit Chromatographiepapier zu erreichen, das mit Dowex-50-Harz imprägniert wurde [108].

Aus ionophoretischen Untersuchungen ${ }^{[109]}$ geht hervor, daß $\mathrm{Pa}(\mathrm{V})$ in $0,6 \mathrm{~N} \mathrm{HCl}+2 \mathrm{~N}$ HF vollständig als Anion

[104] J.Vernois, J. Chromatogr. 1, 52 (1958).

[105] M. Lederer, J. Chromatogr. 1, 172 (1958).

[106] C. Keller, J. Chromatogr. 7, 535 (1962).

[107] Z. Jakovac u. M. Lederer, J. Chromatogr. 1, 291 (1958).

[108] M. Lederer, J. Chromatogr. 2, 210 (1959).

[109] J.Vernois, J. Chromatogr. 2, 155 (1959). vorliegt. Dagegen konnten in Salzsäure sowohl kationische als auch anionische Species nachgewiesen werden [61], wobei der anionische Anteil mit der $\mathrm{HCl}-\mathrm{Kon}$ zentration zunimmt.

\subsection{Spektrophotometrie des Protactiniums}

„Echte“ salzsaure Lösungen des fünfwertigen Protactiniums besitzen bei etwa $210 \mathrm{~m} \mu$ e eine Absorptionsbande mit $\varepsilon \approx 10^{4}\left[35,110^{-113}\right]$. Im Verlaufe des Alterungsprozesses (Hydrolyse) verschwindet diese Bande, gleichzeitig erscheint eine neue Bande bei $260 \mathrm{~m} \mu$, die hydrolysierten Species zugeschrieben wird. Die Absorptionsbande schwefelsaurer $\mathrm{Pa}(\mathrm{V})$-Lösungen verschiebt sich mit zunehmender Schwefelsäurekonzentration von $206 \mathrm{~m} \mu \mathrm{zu} 222 \mathrm{~m} \mu^{[71]}$. Aus der Verschiebung und Intensitätsveränderung der Absorptionsbanden von $\mathrm{Pa}(\mathrm{V})$ in verdünnter Flußsäure schlossen Miranda und Muxart ${ }^{[114]}$ auf die Existenz von mindestens vier $\mathrm{Pa}-\mathrm{F}$ Species.

Die ersten zuverlässigen Angaben über das Absorptionsspektrum von $\mathrm{Pa}(\mathrm{IV})$ stammten von Fried und Hindman ${ }^{[115]}$. Die Autoren lösten reines $\mathrm{PaCl}_{4}$ in sauerstofffreier $1 \mathrm{~N} \mathrm{HCl}$ und erhielten ein Absorptionsspektrum mit Banden bei $276 \mathrm{~m} \mu, 255 \mathrm{~m} \mu$ und $223,8 \mathrm{~m} \mu$, das dem Absorptionsspektrum von Ce(III) sehr ähnelt. Die Absorptionsspektren von $\mathrm{Pa}(\mathrm{IV})$ in anderen Mineralsäuren unterscheiden sich von dem in Salzsäure nur durch die etwas verschiedene Lage und die geringfügig veränderten molaren Extinktionskoeffizienten [116-118]. Wie sich spektrophotometrisch zeigen läßt, sind die wäßrigen Lösungen von $\mathrm{Pa}(\mathrm{IV})$ nur in Anwesenheit eines Überschusses an Reduktionsmittel stabil. Die Geschwindigkeit der Oxidation von $\mathrm{Pa}(\mathrm{IV})$ in flußsaurer Lösung ist in bezug auf die Pa-Konzentration von erster Ordnung. Tageslicht und besonders UV-Licht beschleunigen die Oxidation stark [117].

\subsection{Elektrochemie des Protactiniums}

\subsubsection{Normalpotential, Redoxpotential}

Das Normalpotential der Kette $\mathrm{Pa} \cdot \mathrm{Pa}(\mathrm{V})$ wurde experimentell noch nicht bestimmt. Aus Messungen der Abscheidegeschwindigkeit des Protactiniums aus Losungen verschiedener Konzentrationen $\left(10^{-4}-10^{-11} \mathrm{M}\right)$ auf verschiedene

[110] A.T. Casey u. A. G. Maddock, J. inorg. nuclear Chem. 10 , 58 (1959).

[111] D. Brown, R. G. Wilkths u. A. J. Smuth, J. chem. Soc. (London) $1959,1463$.

[112] R. Guillaumont, R. Muxart, G. Bouissières u. M. Haissinsky, C. R. hebd. Séances Acad. Sci. 248, 3298 (1959).

[113] R. Guillaumont, R. Muxart, G. Bousssières u. M. Haissmsky, J. Chim. physique 57,1019 (1960).

[114] C. F. de Miranda u. R. Muxart, Bull. Soc. chim. France $1964,387$.

[115] S. Fried u. J. C. Hindman, J. Amer. chem Soc. 76,4863 (1954).

[116] D. Brown u. R. G. Wilkins, J. chem. Soc. (London) 1961, 3804 .

[117] M. Hatssinsky, R. Mfuxart u. H. Arapahi, Bull. Soc. chım. France $1961,2248$.

[118] C. F. de Miranda u. R. Muxart, Bull. Soc. chim. France $1964,2174$. 
Metalle - einem erstmals von Hevesy und Paneth angewandten Verfahren - wurde geschlossen [119], daß das Normalpotential der Kette $\mathrm{Pa} / \mathrm{Pa}(\mathrm{V})$ in flußsaurer Lösung bei etwa $-1,0 \mathrm{~V}$ liegt. Das Redoxpotential $\mathrm{Pa}(\mathrm{IV}) \rightarrow \mathrm{Pa}(\mathrm{V})+\mathrm{e}^{-}$ beträgt nach Haissinsky und Pluchet $+0,25 \pm 0,03$ V [120].

\subsubsection{Elektrochemische Abscheidung} auf Metallen

In Spurenmengen scheidet sich $\mathrm{Pa}$ auf zahlreichen $\mathrm{Me}$ talloberfächen sowie auf $\mathrm{PbO}_{2}$ spontan $\mathrm{ab}$, besonders aus flußsauren und schwefelsauren Lösungen [121,122]. Eingehend ist die Abscheidung auf Blei untersucht worden. Sie folgt einer Langmuir-Isotherme. Es wird angenommen, daß sich bei der Abscheidung aus flußsauren Lösungen auf Blei oberflächlich eine Schicht von $\mathrm{PaF}_{4}$ oder $\mathrm{PbPaF}_{7}$ bildet. Die Diffusionskonstante für die $\mathrm{Pa}$ Teilchen bei der Abscheidung beträgt $0,12 \mathrm{~cm}^{2} / \mathrm{Tag}{ }^{[123]}$, während aus polarographischen Untersuchungen ein Wert von $0,62 \mathrm{~cm}^{2} / \mathrm{Tag}{ }^{[124]}$ ermittelt wurde.

Eine quantitative kathodische Abscheidung von Spurenmengen $\mathrm{Pa}$ auf Plättchen aus rostfreiem Stahl ist aus $0,2 \mathrm{M} \mathrm{NH}_{4} \mathrm{~F}-, 0,015 \mathrm{M} \mathrm{NH}_{4} \mathrm{~F}-+0,25 \mathrm{M} \mathrm{NH}_{4} \mathrm{Cl}$ - und $0,02 \mathrm{M} \mathrm{H}_{2} \mathrm{C}_{2} \mathrm{O}_{4^{-}}+0,06 \mathrm{M} \mathrm{HNO}_{3}$-Lösungen bei einer Stromdichte von ca. $160 \mathrm{~mA} / \mathrm{cm}^{2}$ möglich. Es bildet sich eine fest haftende Schicht, auch bei der - empfohlenen - Co-Abscheidung von $50 \mu \mathrm{g} \mathrm{U}_{\text {nat }}{ }^{[125]}$. Andere Auto ren ${ }^{[126-128]}$ erreichten jedoch nur Abscheidungsausbeuten von $80-95 \%$, falls nicht extrem lange Abscheidungszeiten in Kauf genommen wurden.

\subsubsection{Polarographie des Protactiniums}

Bei polarographischen Untersuchungen in $3,8 \mathrm{M} \mathrm{F}^{-}$Lösung ( $\mathrm{pH}=7,2 ; 10^{-3}-10^{-4} \mathrm{M} \mathrm{Pa}$ ) wurden zwei irreversible Wellen bei $-1,29$ und $-1,56 \mathrm{~V}$. bezogen auf eine gesättigte Kalomel-Elektrode, gefunden [124]. Die erste Welle kann der Reduktion des Pa(V)-Fluoro-Komplexes zum entsprechenden Komplex des $\mathrm{Pa}(\mathrm{IV})$ zugeschrieben werden, die zweite Welle wird der Reduktion $\mathrm{Pa}$ (IV) $\rightarrow \mathrm{Pa}$ (III) zugeordnet. Die Höhe der ersten Welle ist der Pa-Konzentration proportional. In $\mathrm{F}^{-}$-freien $\mathrm{Pa}(\mathrm{V})$-Lösungen wurde nur eine irreversible Welle gefunden, die stets die Reduktion $\mathrm{Pa}(\mathrm{V}) \rightarrow \mathrm{Pa}(\mathrm{IV})$ anzeigt.

[119] C. Ferradini, J. Chim. physique 53, 714 (1956).

[120] M. Haissinsky u. E. Pluchet, J. Chım. physique 59, 608 (1962).

[121] M. Camarcat, G. Bouisstères u. M. Hatssmsky, J. Chim. physique 46,153 (1949).

[122] C. Ferradini u. M. Haissinsky, J. Chim. physique 53, 722 (1956).

[123] O. Gautsch, Atompraxis 7, 101 (1961).

[124] C. Ferreira de Mirando u. A. G. Maddock, J. inorg. nuclear Chem. 24, 1623 (1962).

[125] G. Smith u. G. A. Barnett, J. inorg, nuclear Chem. 27, 975 (1965).

[126] H. Shimojima u. J. Takagi, J. inorg. nuclear Chem. 26, 253 (1964).

[127] R. F. Mitchell, Analytıc. Chem. 32, 326 (1960).

[128] J.W. Gofman u. G.T. Seaborg: Nati. Nuclear Energy Series, McGraw-Hill, New York 1954, Bd. IV-14B, S. 1428.

\section{Das Verhalten von Protactinium \\ in Salzschmelzen}

$\mathrm{Pa}(\mathrm{V})$ in Mengen von 1-2 ppb oder 50-75 ppm werden aus einer $\mathrm{LiF}-\mathrm{BeF}_{2}-\mathrm{ThF}_{4}$-Schmelze (67-18-15 Mol- \%) durch Zusatz von $1-2 \%$ festem $\mathrm{BeO}$ bzw. $\mathrm{ThO}_{2}$ ausgefällt. Das $\mathrm{Pa}(\mathrm{V})$ scheidet sich auf der $\mathrm{BeO}-$ bzw. $\mathrm{ThO}_{2}$ Oberfläche ab. Diese Methode kann zur Abtrennung des $233 \mathrm{~Pa}$ und ${ }^{233} \mathrm{U}$ aus dem Brennstoff eines ZweizonenSalzschmelzen-Brutreaktors verwendet werden, wenn man eine parasitäre Neutronenabsorption durch die beiden Nuklide vermeiden will (Erhöhung des Brutgewinns) ${ }^{[129]}$. Bei der Verteilung von $\mathrm{Pa}$ zwischen einer Halogenid- und einer Metallschmelze $\left[\mathrm{CaF}_{2}\right.$ (flüssig)/ Th oder Th, U] reichert sich $\mathrm{Pa}$ in der metallischen Phase an [130], dagegen in den Systemen mit Uran als geschmolzenem Metall und $\mathrm{LiCl}, \mathrm{LiCl} / \mathrm{KCl}, \mathrm{AlCl}_{3}$ oder $\mathrm{AlCl}_{3}$ / $\mathrm{KCl}$ als Halogenid in der Chloridschmelze ${ }^{[131,132]}$.

\section{Die Chemie des Protactiniums in festem Zustand}

\subsection{Protactiniummetall}

Metallisches Protactinium wurde zum ersten Male durch v. Grosse ${ }^{[133]}$ beim Beschuß von Pa-Oxid mit 35-keVElektronen im Hochvakuum oder durch thermische Zersetzung von $\mathrm{PaCl}_{5}$ an einem heißen Draht erhalten. Angaben über Eigenschaften des Metalls wurden jedoch nicht gemacht. Ein reines Metall erhält man bei der Reduktion von $\mathrm{PaF}_{4}$ mit Calcium oder Barium [134-136] bei $1300-1400^{\circ} \mathrm{C}$. Es besitzt ein tetragonal-raumzentriertes Gitter mit $\mathrm{a}=3,929 \AA$ und $\mathrm{c}=3,241 \AA[135,137]$. Wahrscheinlich existieren noch zwei weitere Modifikationen, eine kubisch-raumzentrierte oberhalb $1170^{\circ} \mathrm{C}$ und eine niedriger symmetrische Modifikation, die durch rasches Abschrecken von $1580^{\circ} \mathrm{C}$ auf Zimmertemperatur erhalten wird. Der Schmelzpunkt liegt mit $1560^{\circ} \mathrm{C}$ zwischen den Werten für die Nachbarelemente Thorium $\left(1750^{\circ} \mathrm{C}\right)$ und Uran $\left(1132^{\circ} \mathrm{C}\right)$. Pa-Metall bildet kein Amalgam ${ }^{[1381}$. Die magnetische Suszeptibilität $\alpha=$ $270 \times 10^{-6}$ cgs-Einheiten) ist - wie beim Tantal - praktisch temperaturunabhängig.

Mit $6 \mathrm{~N} \mathrm{HCl}$ reagiert Pa-Metall anfangs sehr stürmisch, die Auflösung hört nach kurzer Zeit jedoch wieder auf,

[129] J. H. Shaffer, W. R. Grimes, G. M. Watson, D. R. Cuneo, J. E. Strain u. M. J. Kelly, Nuclear Sci. Engng. 18, 177 (1964).

[130] G. E. Brand, E.W. Murbach u. A. G. Buyers, Nuclear Sci. Engng. 5, 157 (1959).

[131] P. Chiotto u. S. J. S. Parry, Iowa State Iniversity, Report JS-286 (1961).

[132] R. H. Moore u. W. L. Lyon, General Electric Co., Hanford Products Operation, Report HW-59147 (1959).

[133] A. v. Grosse u. M. Agruss, J. Amer. chem. Soc. 56, 2200 (1934).

[134] P. A. Sellers, S. M. Fried, R. E. Elson u. W. H. Zachariasen, J. Amer. chem. Soc. 76, 5935 (1954).

[135] B. B. Cunninghan, Colloque International sur la PhysicoChimie du Protactinium, Orsay/Frankreich, Juli 1965.

[136] J. A. C. Marples, Acta crystallogr. 18, 815 (1965).

[137] W. H. Zachariasen, Acta crystallogr. 5, 17 (1952).

[138] G. Bouissières u. Y.Lagoux, Bull. Soc. chım. France 1965, 386. 
was auf die Bildung einer Oxid-Oberflächenschicht zurückgeführt wird [135]. Wasserstoff bildet bei $250-300^{\circ} \mathrm{C}$ mit Pa-Metall ein Hydrid $\mathrm{PaH}_{3}$ (kubisch, $\mathrm{a}=6,648 \AA$ ), das mit $\mathrm{UH}_{3}$ isostrukturell ist ${ }^{[134]}$.

\subsection{Verbindungen des Protactiniums}

\subsubsection{Binäre Verbindungen}

Beim Eindampfen einer flußsauren, wäßrigen Lösung von $\mathrm{Pa}(\mathrm{V})$ erhält man $\mathrm{PaF}_{5} \cdot 2 \mathrm{H}_{2} \mathrm{O}^{[139]}$, das sich oberhalb $160^{\circ} \mathrm{C}$ zu dem Oxidfluorid $\mathrm{Pa}_{2} \mathrm{OF}_{8}$ (kubisch raumzentriert, $\mathrm{a}=8,4065 \AA$, isotyp mit $\mathrm{U}_{2} \mathrm{~F}_{9}$ ) zersetzt und wahrscheinlich eine $\mathrm{F}_{4} \mathrm{~Pa}-\mathrm{O}-\mathrm{PaF}_{4}-\mathrm{Brücke}$ enthält. $\mathrm{Pa}_{2} \mathrm{OF}_{8}$ wird auch bei der direkten Fluorierung von $\mathrm{Pa}_{2} \mathrm{O}_{5}$ bei $550^{\circ} \mathrm{C}$ sowie der Hydrolyse von $\mathrm{PaF}_{5}$ gebildet. Reines $\mathrm{PaF}_{5}$ erhält man nur bei der Fluorierung von $\mathrm{PaF}_{4}$ mit reinstem Fluor. Das tetragonal mit der Struktur von $\beta-\mathrm{UF}_{5}$ kristallisierende $\mathrm{PaF}_{5}(\mathrm{a}=11,53 \AA, \mathrm{c}=$ $5,19 \AA)$ ist weniger flüchtig als $\mathrm{VF}_{5}, \mathrm{NbF}_{5}$ und $\mathrm{TaF}_{5}$, kann jedoch oberhalb $500^{\circ} \mathrm{C}$ bei $10^{-5}-10^{-6}$ Torr sublimiert werden. Durch Reaktion von $\mathrm{Pa}_{2} \mathrm{O}_{5}$ mit $\mathrm{H}_{2} / \mathrm{HF}$ oberhalb $500^{\circ} \mathrm{C}$ lassen sich $\mathrm{Pa}_{4} \mathrm{~F}_{17}$ (kubisch raumzentriert, $\mathfrak{a}=8,507 \AA$ ) und $\mathrm{PaF}_{4}$ (monoklin, isotyp mit $\mathrm{ThF}_{4}$ und $\mathrm{UF}_{4}$ ) herstellen $[139,140] . \mathrm{PaF}_{4}$ ist im Gegensatz zu $\mathrm{PaF}_{5}$ in verdünnter Flußsäure nicht löslich, es löst sich dagegen in $15 \mathrm{M} \mathrm{NH}_{4} \mathrm{~F}-\mathrm{Lösungen} \mathrm{[117].}$

Beim Zusatz von Fluoridionen zu einer schwefelsauren $\mathrm{Pa}^{4+}$-Lösung erhält man $\mathrm{PaF}_{2} \mathrm{SO}_{4} \cdot 2 \mathrm{H}_{2} \mathrm{O}{ }^{[140]}$. Die Darstellung der entsprechenden Uran-Verbindung läßt eine Analogie zwischen den Ionen $\mathrm{UF}_{2}^{2+}$ und $\mathrm{PaF}_{2}^{2+}$ und dem stabilen $\mathrm{UO}_{2}^{2+-}$-Ion vermuten.

$\mathrm{PaCl}_{5}$ erhält man am einfachsten durch Reaktion von $\mathrm{Pa}_{2} \mathrm{O}_{5}$ mit einem mit $\mathrm{CCl}_{4}$-Dampf gesättigten Chlorstrom bei $300-400^{\circ} \mathrm{C}[141]$. Sein Schmelzpunkt beträgt $300^{\circ} \mathrm{C}$, im Vakuum sublimiert es jedoch bereits oberhalb $160^{\circ} \mathrm{C}$. Die leichte Flüchtigkeit des $\mathrm{PaCl}_{5} \mathrm{kann}$ für verschiedene Trennungen benutzt werden, z. B. für die Gewinnung von ${ }^{233} \mathrm{~Pa}$ aus neutronenbestrahltem $\mathrm{ThCl}_{4}{ }^{[142]} . \mathrm{PaCl}_{5}$ kristallisiert in einem monoklinen Gitter $(\mathrm{a}=10,25 \AA, \mathrm{b}=12,31 \AA, \mathrm{c}=8,82 \AA, \beta=$ $\left.111,8^{\circ} \mathrm{C}\right)^{[143]}$ und weist - wic alle binären Halogenide $\mathrm{PaX}_{5}$ - andere Struktur auf als die entsprechenden $\mathrm{Nb}$ und Ta-Pentahalogenide. Orangerotes $\mathrm{PaBr}_{5}(\mathrm{Fp}=$ $317^{\circ} \mathrm{C}$ ) und schwarzes $\mathrm{PaJ}_{5}$ erhält man durch Reaktion von $\mathrm{Pa}_{2} \mathrm{O}_{5}$ mit $\mathrm{AlBr}_{3}$ bzw. $\mathrm{AlJ}_{3}$. Die Reindarstellung ist durch Vakuum-Sublimation möglich, allerdings zersetzt sich $\mathrm{PaJ}_{5}$ bereits oberhalb $300^{\circ} \mathrm{C}$ unter Jodabspaltung. Beide Salze besitzen orthorhombische Struktur $\left(\mathrm{PaBr}_{5}\right.$ : $\mathrm{a}=7,25 \AA, \mathrm{b}=12,12 \AA, \mathrm{c}=9,13 \AA ; \mathrm{PaJ}_{5}: \mathrm{a}=7,22 \AA$, $\mathrm{b}=21,2 \AA, \mathrm{c}=6,85 \AA$ ).

\section{[139] L. Stein, Inorg. Chem. 3, 995 (1964).}

[140] L. Stein, Colloque International sur La Physıco-Chımı du Protactinium, Orsay/Frankreich, Juli 1965.

[141] J. Flegenheimer, Thesis, Cambridge (1959).

[142] J. Mérinis, Y. Legoux u. G. Boussicres, Colloque International sur la Physico-Chimie du Protactınium, Orsay/Frankreich, Juli 1965.

[143] Referiert in: D. Brown u. A. G. Maddock, Quart. Rev. 1963, 289.
Durch Reaktion von $\mathrm{PaO}_{2}$ mit $\mathrm{CCl}_{4}$ oder durch Reduktion von $\mathrm{PaCl}_{5}$ mit Wasserstoff bei $400-500^{\circ} \mathrm{C}$ in einem abgeschlossenen System erhält man $\mathrm{PaCl}_{4}$ (tetragonal $\mathrm{a}=8,377 \AA, \mathrm{c}=7,482 \AA$ ), isotyp mit $\mathrm{UCl}_{4}[134]$.

\subsubsection{Polynäre Verbindungen}

Durch Umsetzung von $\mathrm{PaF}_{4}$ mit einem Überschuß an $\mathrm{NH}_{4} \mathrm{~F}$ läßt sich das mit der entsprechenden $\mathrm{U}_{-}, \mathrm{Np}-, \mathrm{Pu}-$ und Am-Verbindung [144-146] isotype $\left(\mathrm{NH}_{4}\right)_{4} \mathrm{PaF}_{8}$ darstellen [147]. Bei der Festkörperreaktion von $\mathrm{RbF}$ mit $\mathrm{PaF}_{4}$ erhält man die Verbindung $7 \mathrm{RbF}^{6} 6 \mathrm{PaF}_{4}$ (orthorhombisch, $\left.\mathrm{a}=9,587 \AA, \alpha=107^{\circ} 9^{\prime}\right)^{[147 \mathrm{a}]}$, die mit den analogen Th-[148], U-[149], $\mathrm{Np}$ - und $\mathrm{Pu}$-Verbindungen ${ }^{[150]}$ isotyp ist.

Doppelfluoride des fünfwertigen Protactiniums lassen sich sowohl aus wäBriger Lösung als auch durch Fluorierung eines $\mathrm{MF} / \mathrm{PaF}_{4}$-Gemisches herstellen. Das schwerlösliche $\mathrm{K}_{2} \mathrm{PaF}_{7}$, welches übrigens die erste reine Verbindung des Protactiniums war, wurde zuerst durch v. Grosse [151] erhalten. Oberhalb $130^{\circ} \mathrm{C}$ zersetzt es sich in $\mathrm{KPaF}_{6}$ und $\mathrm{KF}{ }^{[152]}$. Die Struktur von $\mathrm{K}_{2} \mathrm{PaF}_{7}$ wurde erst kürzlich von Brown und Smith [152a] aufgeklärt. $\mathrm{K}_{2} \mathrm{PaF}_{7}$ kristallisiert monoklin mit $\mathrm{a}=13,94, \mathrm{~b}=6,76$, $c=8,24 \AA ; \beta=125,5^{\circ}$ und $Z=4$ (Raumgruppe $C 2 / \mathrm{c}=$ $\mathrm{C}_{2 \mathrm{~h}}^{6}$ ). Jedes $\mathrm{Pa}$-Atom ist von neun Fluoratomen in einer Art umgeben, die am besten als trigonales Prisma beschrieben werden kann, wobei weiterhin in der Äquatorialebene zusätzlich drei Fluoratome hinzugefügt werden. Diese $\mathrm{PaF}_{9}$-Gruppen sind parallel [001] über zwei Fluor-Brückenglieder zu unendlichen Ketten verknüpft. Die interatomaren $\mathrm{Pa}-\mathrm{F}$-Bindungsabstände liegen zwischen $2,13 \AA$ und $2,46 \AA$. Je nach dem Verhältnis $\mathrm{M}^{\mathrm{I}}: \mathrm{Pa}$ lassen sich aus flußsauren Lösungen von $\mathrm{Pa}(\mathrm{V}) \mathrm{kom}-$ plexe Fluoride der Zusammensetzung $\mathrm{M}^{\mathrm{I}} \mathrm{PaF}_{6}, \mathrm{M}^{\mathrm{II}} \mathrm{PaF}_{7}$ und $\mathrm{M}_{3}^{1} \mathrm{PaF}_{8}$ darstellen [153-158]. Die Mehrzahl dieser

[144] R. A. Penteman, F. H. Kruse, R. S. George u. J. S. Coleman, Inorg. Chem. 3, 309 (1964).

[145] R. Benz, R. M. Donglass, F. H. Kruse u. R. A. Penteman, Inorg. Chem. 2, 799 (1963).

[146] L. B. Asprey u. R. A. Penneman, Inorg. Chem. 1,134 (1962) [147] L. B. Asprey u. R. A. Penteman, Colloque International sur la Physico-Chimie du Protactınum, Orsay'Frankreich, Juli 1965.

[147a] L. B. Asprey, F. H. Kruse u. R. A. Penneman, J. Amer. chem. Soc. 87,3518 (1965).

[148] R. E.Thoma, H. Insley, B. S. Landau, H. A. Friedman u. WV. R. Grimes, J. Amer. chem. Soc. 63, 1266 (1959).

[149] C. J. Barton, H. A. Friedman, W. R. Grimes, H. Insley, R. E. Moore u. R. E. Thoma, J. Amer. ceram. Soc. 41, 63 (1958). [150] C. Keller u. H. Schmutz, unveröffentlicht.

[151] A.v. Grosse, J. Amer. chem. Soc. 56, 2501 (1934).

[152] C. Ferreira de Mranda, Colloque International sur la Physıco-Chimie du Protactınıum, Orsay/Frankresch, Juli 1965 [152a] D. Brown u. A.J.Smith, persónl. Mitteilung(Oktober 1965) [153] D. Brown, Colloque International sur la Physico-Chimie du Protactinum. Orsay/Frankreich, Jull 1965.

[154] M. N. Kukhsh, J. Flegenheiner, F. M. Hall, A. G. Maddock u. C. Ferreira de Miranda, Colloque International sur la PhysicoChimie du Protactinium, Orsay! Frankreich, Julı 1965.

[155] D. Brown u. J. E. Easey, Nature (London) 205, 589 (1965). [156] L. B. Asprey u. R. A. Penneman, Scrence 145, 924 (1964). [157] O.L. Keller u. A. Chetham-Strode, Colloque International sur la Physico-Chmie du Protactınıum, Orsay/Frankreich, Juli 1965. [158] L. B. Asprey, R. A. Penneman u. F. H. Kruse, Colloque International sur la Physico-Chimie du Protactinium. Orsay/ Frankreich, Juh 1965. 
Doppelfluoride ist isotyp mit der entsprechenden Uranverbindung, aber nicht mit den Verbindungen des Niobs und Tantals, bei denen der Verbindungstyp $\mathrm{M}_{3} \mathrm{XF}_{8}$ überhaupt fehlt. Allerdings sind bei den Doppelfluoriden nur von $\mathrm{Li}_{3} \mathrm{PaF}_{8}$ (tetragonal, Raumgruppe $\mathrm{D}_{4 \mathrm{~h}}^{6}, \mathrm{a}=10,386 \AA$, $c=10,89 \AA, Z=8$ ), $\mathrm{Na}_{3} \mathrm{PaF}_{8}$ (tetragonal, Raumgruppe $\mathrm{D}_{4 \mathrm{~h}}^{17}, \mathrm{a}=5,487 \AA, \mathrm{c}=10,89 \AA, \mathrm{Z}=2$ ) und $\mathrm{MPaF}_{6}$ (orthorhombisch für $\mathrm{M}=\mathrm{K}, \mathrm{Rb}, \mathrm{Cs} ; \mathrm{KPaF}_{6}: \mathrm{a}=5,64 \AA, \mathrm{b}=$ $5,77 \AA$ und $c=3,99 \AA$ ) nähere Strukturdaten bekannt $[155]$.

Frisch bereitetes $\mathrm{Pa}_{2} \mathrm{O}_{5}$ aq löst sich bei Zimmertemperatur in $\mathrm{SOCl}_{2}$. Nach Abdestillieren des überschüssigen $\mathrm{SOCl}_{2}$ bleibt $\mathrm{SO}\left(\mathrm{PaCl}_{6}\right)_{2}$ als Rückstand, im Gegensatz zu Nb und $\mathrm{Ta}$, von denen man die wasserfreien Chloride erhält $[153,159], \mathrm{SO}\left(\mathrm{PaCl}_{6}\right)_{2}$ zersetzt sich im Vakuum oberhalb $150^{\circ} \mathrm{C}$. Durch Zugabe von $\mathrm{MCl}\left(\mathrm{M}^{+}=\mathrm{Cs}^{+}\right.$, $\left[\left(\mathrm{CH}_{3}\right)_{4} \mathrm{~N}\right]^{+}$oder $\left.\left[\left(\mathrm{C}_{6} \mathrm{H}_{5}\right)_{4} \mathrm{As}\right]^{+}\right)$zur Lösung von $\mathrm{SO}\left(\mathrm{PaCl}_{6}\right)_{2}$ in $\mathrm{SOCl}_{2}$ erhält man Hexachlorokomplexe und Octachlorokomplexe $\mathrm{MPaCl}_{6}$ bzw. $\mathrm{M}_{3} \mathrm{PaCl}_{8}$, letztere jedoch nicht mit $\left[\left(\mathrm{C}_{6} \mathrm{H}_{5}\right)_{4} \mathrm{As}\right] \mathrm{Cl}$. Analoge Doppelchloride lassen sich auch von $\mathrm{U}(\mathrm{V})$ darstellen, von $\mathrm{Nb}$ und Ta existieren jedoch nur Hexachloro-Verbindungen. $\mathrm{PaCl}_{5}$ - in Acetonitril + Dichlormethan gelöst - bildet mit Phosphinoxiden $\mathrm{R}_{3} \mathrm{PO}$ ein Addukt $\mathrm{PaCl}_{5} \cdot \mathrm{R}_{3} \mathrm{PO}$, welches bedeutend stabiler ist als das reine Pentachlorid. Gibt man zur Lösung von $\mathrm{PaBr}_{5}, \mathrm{TaBr}_{5}$ oder $\mathrm{NbBr}_{5}$ in Acetonitril Tetraalkylammoniumbromid, so erhält man nach Abdampfen des Lösungsmittels Doppelbromide des Typs $\mathrm{M}^{\mathrm{I}} \mathrm{M}^{\mathrm{V}} \mathrm{Br}_{6}{ }^{[153]}$.

\subsection{Sauerstoffverbindungen des Protactiniums}

\subsubsection{Das System $\mathrm{Pa}-\mathrm{O}$}

Neben den stöchiometrischen Oxiden des vier- und fünfwertigen Protactiniums existieren noch vier Oxidphasen mit variablem Sauerstoffgehalt (Tabelle 5), die sowohl durch partielle Oxidation von $\mathrm{PaO}_{2}$ mit Sauerstoff als auch durch partielle Reduktion von $\mathrm{Pa}_{2} \mathrm{O}_{5}$ mit Wasserstoff dargestellt werden können. Die Strukturen dieser Verbindungen lassen sich weitgehend vom Fluoritgitter ableiten, was die nahe Verwandtschaft des $\mathrm{PaO}_{2+\mathrm{x}} \mathrm{zu}$ den niederen Uranoxiden zeigt. Infolge der - im Vergleich zu $\mathrm{UO}_{2}$ - größeren $\mathrm{PaO}_{2}$-Elementarzelle ist ein erhöhter Einbau von Sauerstoff auf Zwischengitterplätze des Fluoritgitters möglich, ohne daß das Fluoritgitter ganz geändert wird: im System $\mathrm{PaO}_{2} / \mathrm{O}_{2}$ bis $\mathrm{PaO}_{2,50}$, im System $\mathrm{UO}_{2} / \mathrm{O}_{2}$ nur bis $\mathrm{UO}_{2,35}$.

Nach Zugabe von $\mathrm{H}_{2} \mathrm{O}_{2}$ zu einer verdünnt schwefelsauren $\mathrm{Pa}(\mathrm{V})$-Lösung erhält man einen Niederschlag von amorphem und leicht zersetzlichem Pa-Peroxid $\mathrm{Pa}_{2} \mathrm{O}_{9} \cdot 3 \mathrm{H}_{2} \mathrm{O}[163,164]$, das unterhalb $200^{\circ} \mathrm{C}$ in Protactiniumoxid-Monohydrat übergeht, welches seinerseits bis $280^{\circ} \mathrm{C}$ stabil ist.

\subsubsection{Polynäre Oxide des Protactiniums}

$\mathrm{Pa}_{2} \mathrm{O}_{5}$ verhält sich amphoter, es löst sich - wie ein basisches Oxid - in geschmolzenen Hydrogensulfaten, reagiert aber auch als saures Oxid mit Alkali-und Erdalkalioxiden. Aus Festkörperreaktionen von $\mathrm{PaO}_{2}$ und $\mathrm{Pa}_{2} \mathrm{O}_{5}$ mit Oxiden anderer Elemente ist zu schließen, daß sich Protactinium wie ein ,echtes“ Actinidenelement verhält (Tabelle 6). So bildet $\mathrm{PaO}_{2}$ mit $\mathrm{GeO}_{2}$ oder $\mathrm{SiO}_{2}$ ternäre Oxide des Typs $\mathrm{Pa}^{\mathrm{III}} \mathrm{X}^{\mathrm{IV}} \mathrm{O}_{4}$, die auch von den anderen vierwertigen Actinidenelementen, aber nicht von $\mathrm{Nb}$ (IV) und Ta(IV) bekannt sind. $\mathrm{Pa}^{\mathrm{V}} \mathrm{Nb}_{3} \mathrm{O}_{10}$ entspricht weiterhin vollkommen den Verbindungen $\mathrm{UNb}_{3} \mathrm{O}_{10,67}$ und $\mathrm{NpNb}_{3} \mathrm{O}_{9}, 5$, trotz unterschiedlicher Sauerstoffzahl. Mit den Sesquioxiden der Lanthaniden und Actiniden rea* giert $\mathrm{Pa}_{2} \mathrm{O}_{5}$ zu Doppeloxiden des Typs $\left(\mathrm{M}_{0.5}^{\mathrm{II}}, \mathrm{Pa}_{0,5}^{\mathrm{V}}\right) \mathrm{O}_{2}$ mit Fluoritstruktur und statistischer Verteilung der Schwermetallionen auf die $\mathrm{Ca}^{2+-P l a ̈ t z e}$ des $\mathrm{CaF}_{2}$-Gitters. Ähnlich wie alle Actinidendioxide nehmen die $\mathrm{M}(\mathrm{III}) / \mathrm{Pa}(\mathrm{V})$-Doppeloxide weiteres $\mathrm{MO}_{1,5}$ oder $\mathrm{PaO}_{2,5}$ in fester Lösung auf, die Löslichkeit von $\mathrm{MO}_{1,5}$ im

Tabelle 5. Phasenverhảltnisse im System $\mathrm{Pa}-\mathrm{O}$.

\begin{tabular}{|c|c|c|c|c|c|c|c|}
\hline \multirow{2}{*}{ Zusammensetzung } & \multirow{2}{*}{ Struktur } & \multicolumn{4}{|c|}{ Gitterkonstanten } & \multirow{2}{*}{$\begin{array}{l}\text { Existenz- } \\
\text { bereich }\left[{ }^{\circ} \mathrm{C}\right]\end{array}$} & \multirow{2}{*}{ Lit. } \\
\hline & & $\mathrm{a}[\AA]$ & $\mathrm{b}[\AA]$ & $c[\AA]$ & $\alpha$ & & \\
\hline $\begin{array}{l}\mathrm{PaO}(?) \\
\mathrm{PaO}_{2} \\
\mathrm{PaO}_{2,18}-\mathrm{PaO}_{2,2 t} \\
\mathrm{PaO}_{2,33} \\
\mathrm{PaO}_{2,40}-\mathrm{PaO}_{2,42} \\
\mathrm{PaO}_{2,42}-\mathrm{PaO}_{2,44} \\
\mathrm{~Pa}_{2} \mathrm{O}_{5}\end{array}$ & $\begin{array}{l}\text { kubisch }(\mathrm{NaCl}) \\
\text { kubisch }\left(\mathrm{CaF}_{2}\right) \\
\text { kubisch } \\
\text { tetragonal } \\
\text { tetragonal } \\
\text { rhomboedrisch } \\
\text { kubisch }\left(\mathrm{CaF}_{2}\right) \\
\text { tetragonal } \\
\text { hexagonal } \\
\text { rhomboedrisch } \\
\text { orthorhombisch }\end{array}$ & $\begin{array}{l}4,961 \\
5,505 \\
5,473 \\
5,425 \\
5,480 \\
5,449 \\
5,446 \\
5,429 \\
3,817 \\
5,424 \\
6,92\end{array}$ & 4,02 & $\begin{array}{l}5,568 \\
5,416 \\
5,503 \\
13,22 \\
4,18\end{array}$ & $89,76^{\circ}$ & $\begin{array}{l}700 \\
1000 \\
1000-1200 \\
1240-1400 \\
? \\
200-280\end{array}$ & $\begin{array}{l}{[153]} \\
{[153]} \\
{[160]} \\
{[160]} \\
{[160]} \\
{[160]} \\
{[153,161]} \\
{[160,161]} \\
{[160,161]} \\
{[160]} \\
{[153,160-162]} \\
{[163]}\end{array}$ \\
\hline
\end{tabular}

$\overrightarrow{[159] K . W .}$ Bagnall u. D. Brown, J. chem. Soc. (London) 1964, 3021.

[160] L. E. J. Roberts u. A. J. Walter, Colloque International sur la Physico-Chimı du Protactınium, Orsay/Frankreich, Juli 1965.

[161] T. Stchouzkoy, H. Pézerat, G. Bouissières u. R. Muxart, C. R. hebd. Séances Acad. Sci. 259, 3016 (1964).

[162] H. Kirby, J. inorg. nuclear Chem. 18, 8 (1961).

[163] T. Stchouzkoy, H. Pezerat u. R. Muxart, Colloque International sur la Physico-Chimie du Protactınium, Orsay/Frankreich, Juli 1965.
$\left(\mathrm{M}_{0,5}, \mathrm{~Pa}_{0,5}\right) \mathrm{O}_{2}$-Wirtsgitter ist hierbei eine Funktion des Ionenradius von $\mathrm{MO}_{\mathrm{I}, 5}$ (Abb. 6). Die Gitterkonstanten der festen Lösungen ändern sich linear mit der Menge des in $\left(\mathrm{M}_{0,5}, \mathrm{~Pa}_{0,5}\right) \mathrm{O}_{2}$ gelösten $\mathrm{MO}_{1,5}$. Mit steigender Temperatur nimmt die Löslichkeit zu. Ähnlich wie im System $\mathrm{Li}_{2} \mathrm{O} / \mathrm{Pa}_{2} \mathrm{O}_{5}{ }^{[173]}$ existiert auch im System [164] T. Stchouzkoy u, H. Pezerat, Bull. Soc. chım. France 1962, 2176 . 


\begin{tabular}{|c|c|c|c|c|c|c|c|}
\hline \multirow{2}{*}{ Verbindung } & \multirow{2}{*}{ Herstellungsbedingungen } & \multirow{2}{*}{ Struktur } & \multicolumn{4}{|c|}{ Gitterkonstanten } & \multirow{2}{*}{$\begin{array}{l}\text { Isostrukturelle } \\
\text { Verbindungen } \\
\text { mit }\end{array}$} \\
\hline & & & $\mathbf{a}[\AA]$ & $\mathrm{b}[\boldsymbol{A}]$ & $c[\AA]$ & $\beta$ & \\
\hline $\mathrm{LiPaO}_{3}$ & $\mathrm{LiO}+\mathrm{Pa}_{2} \mathrm{O}_{5} / 600^{\circ} \mathrm{C} / 2<8 \mathrm{~h} / \mathrm{O}_{2}$ & unbekannt & & & & & \\
\hline $\mathrm{Li}_{3} \mathrm{PaO}_{4}$ & & tetragonal; $\mathrm{L}_{3} \mathrm{UO}_{4}$ & 4,52 & & 8,48 & & $\mathrm{U}, \mathrm{Np}, \mathrm{Pu}, \mathrm{Am}$ \\
\hline $\mathrm{Li}_{7} \mathrm{PaO}_{6}$ & & hexagonal; $\mathrm{Li}_{7} \mathrm{~B}_{3} \mathrm{O}_{6}$ & 5,55 & & 15,84 & & $\mathrm{U}, \mathrm{Np}, \mathrm{Pu}, \mathrm{Am}$ \\
\hline$(2-4) \mathrm{Li}_{2} \mathrm{O} \cdot \mathrm{Pa}_{2} \mathrm{O}_{5}$ & $\mathrm{Li}_{2} \mathrm{CO}_{3}+\mathrm{Pa}_{2} \mathrm{O}_{5}$ & kubisch, Fluoritphase & & & & & \\
\hline$(2-4) \mathrm{Na}_{2} \mathrm{O} \cdot \mathrm{Pa}_{2} \mathrm{O}_{5}$ & $\mathrm{Na}_{2} \mathrm{CO}_{3}+\mathrm{Pa}_{2} \mathrm{O}_{5}$ & & & & & & \\
\hline $\mathrm{NaPaO}_{3}$ & $\mathrm{Na}_{2} \mathrm{O}_{2}+\mathrm{Pa}_{2} \mathrm{O}_{5} / 500^{\circ} \mathrm{C} / 2 \times 8 \mathrm{~h} / \mathrm{Ar}$ & orthorhombisch; $\mathrm{GdFeO}_{3}$ & 5,82 & 5,97 & 8,36 & & $\mathbf{U}$ \\
\hline $\mathrm{Na}_{3} \mathrm{PaO}_{4}$ & & tetragonal; $\mathrm{L}_{3} \mathrm{SbO}_{4}$ & 6,68 & & 9,60 & & \\
\hline $\mathrm{KPaO}_{3}$ & $\mathrm{~K}_{2} \mathrm{CO}_{3}+\mathrm{Pa}_{2} \mathrm{O}_{5} / 600^{\circ} \mathrm{C} / 2<8 \mathrm{~h} / \mathrm{O}_{2}$ & kubisch; $\mathrm{CaT}_{1} \mathrm{O}_{3}$ & 4,341 & & & & $U$ \\
\hline $\mathrm{RbPaO}_{3}$ & $\mathrm{Rb}_{2} \mathrm{CO}_{3}+\mathrm{Pa}_{2} \mathrm{O}_{5} / 500^{\circ} \mathrm{C} / 2 \times 8 \mathrm{~h} / \mathrm{O}_{2}$ & & 4,368 & & & & $\mathrm{U}$ \\
\hline $\mathrm{CsPaO}_{3}$ & $\mathrm{CsCO}_{3}+\mathrm{Pa}_{2} \mathrm{O}_{5} / 500^{\circ} \mathrm{C} / 2 \times 8 \mathrm{~h} / \mathrm{O}_{2}$ & unbekannt & & & & & \\
\hline $\mathrm{BaPaO}_{3}[a]$ & $\mathrm{BaO}+\mathrm{PaO}_{2} / 1200^{\circ} \mathrm{C}-8 \mathrm{~h} /$ Vakuum & kubisch; $\mathrm{CaTiO}_{3}$ & 4,45 & & & & $\mathrm{U}, \mathrm{Np}, \mathrm{Pu}, \mathrm{Am}$ \\
\hline $\mathrm{SrPaO}_{3}[\mathrm{a}]$ & $\mathrm{SrO}+\mathrm{PaO}_{2} / 1200^{\circ} \mathrm{C} / 8 \mathrm{~h} /$ Vakuum & unbekannt & & & & & \\
\hline $\begin{array}{l}\mathrm{Ba}\left(\mathrm{Ba}_{0,5}, \mathrm{~Pa}_{0,5}\right) \mathrm{O}_{2,75} \\
\mathrm{GaPaO}_{4}\end{array}$ & $\begin{array}{l}\mathrm{BaO}+\mathrm{Pa}_{2} \mathrm{O}_{5} / 1350^{\circ} \mathrm{C} / 2 \times 6 \mathrm{~h} / \mathrm{O}_{2} \\
\mathrm{Ga}_{2} \mathrm{O}_{3}+\mathrm{Pa}_{2} \mathrm{O}_{5} / 1200^{\circ} \mathrm{C} / 8 \mathrm{~h} / \mathrm{O}_{2}\end{array}$ & $\begin{array}{l}\text { kubisch, } \mathrm{Ba}_{3} \mathrm{WO}_{6} \\
\text { unbekannt }\end{array}$ & 8,932 & & & & $\begin{array}{l}\mathrm{Nb}, \mathrm{Ta}, \mathrm{U} \\
M I I I=S . E ., Y,[b]\end{array}$ \\
\hline$\left(\mathrm{La}_{0,5}, \mathrm{~Pa}_{0,5}\right) \mathrm{O}_{2}$ & $\mathrm{La}_{2} \mathrm{O}_{3}+\mathrm{Pa}_{2} \mathrm{O}_{5} / 1100^{\circ} \mathrm{C} / 2 \times 8 \mathrm{~h} / \mathrm{O}_{2}$ & kubisch, $\mathrm{CaF}_{2}$ & 5,525 & & & & $\mathrm{Sc}, \mathrm{In}, \mathrm{Pu}, \mathrm{Cm}$ \\
\hline $\mathrm{Ba}\left(\mathrm{La}_{0}, 5, \mathrm{~Pa}_{0,5}\right) \mathrm{O}_{3}$ & $\mathrm{BaO}+\left(\mathrm{La}_{0,5}, \mathrm{~Pa}_{0,5}\right) \mathrm{O}_{2} / 1350^{\circ} \mathrm{C} / 12 \mathrm{~h} / \mathrm{O}_{2}$ & kubisch, $\mathrm{Ba}_{3} \mathrm{WO}_{6}$ & 8,885 & & & & $\begin{array}{l}\text { MIII }=S . E ., Y \\
S c, I n, P u\end{array}$ \\
\hline$\alpha-\mathrm{PaGeO}_{4}$ & $\mathrm{PaO}_{2}+\mathrm{GeO}_{2} / 1100^{\circ} \mathrm{C} / 2 \times 12 \mathrm{~h} /$ Vakuum & tetragonal, $\mathrm{CaWO}_{4}$ & 5,106 & & 11,38 & & \\
\hline$\beta-\mathrm{PaGeO}_{4}[\mathrm{a}]$ & $\begin{array}{l}\alpha-\mathrm{PaGeO}_{4} \underset{\mathrm{Pad}}{\text { hydrothermal } / 230^{\circ} \mathrm{C} / 5 \mathrm{~d}} \\
\end{array}$ & tetragonal, $\mathrm{ZrS}_{1} \mathrm{O}_{4}$ & 7,068 & & 6,509 & & $\mathrm{Th}, \mathrm{U}, \mathrm{Np}$ \\
\hline$\alpha-\mathrm{PaSiO}_{4}[\mathrm{a}]$ & $\begin{array}{l}\text { hydrothermal/230d/5d } \\
11200^{\circ} \mathrm{C}\end{array}$ & tetragonal; $\mathrm{ZrS}_{1} \mathrm{O}_{4}$ & 7,068 & & 6,288 & & $\mathrm{Th}, \mathrm{U}, \mathrm{Np}, \mathrm{Pu}, \mathrm{Am}$ \\
\hline$\beta-\mathrm{PaSiO}_{4}[\mathrm{a}]$ & $\begin{array}{l}\alpha-\mathrm{PaS}_{1} \mathrm{O}_{4} \underset{\mathrm{PaO}}{\mathrm{PaO}}+\mathrm{SiO}_{2} / 1250 \% / 8 \mathrm{~h} / \text { Vakuum }\end{array}$ & monoklin; $\mathrm{CePO}_{4}$ & 6,76 & 6,92 & 6,45 & $104^{\circ} 50^{\prime}$ & Th \\
\hline $\mathrm{Pa}_{2} \mathrm{O}_{5} / \mathrm{ThO}_{2}$ & $\begin{array}{l}\mathrm{Pa}_{2} \mathrm{O}_{\mathrm{S}}+\mathrm{ThO}_{2} / 1100^{\circ} \mathrm{C} / 8 \mathrm{~h} / \mathrm{O}_{2} \\
(\mathrm{~Pa}, \mathrm{Th}) \mathrm{O}_{2}-\mathrm{O}_{2} / 400^{\circ} \mathrm{C}\end{array}$ & kubisch, Fluoritphase & & & & & \\
\hline $\mathrm{PaO}_{2} \cdot 2 \mathrm{Nb}_{2} \mathrm{O}_{5}[\mathrm{a}]$ & $\mathrm{PaO}_{2}+\mathrm{Nb}_{2} \mathrm{O}_{5} / 1200^{\circ} \mathrm{C} / 8 \mathrm{~h} /$ Vakuum & tetragonal, $\mathrm{Th}_{0,25} \mathrm{NbO}_{3}$ & 7,76 & & 7,81 & & $\mathrm{U}, \mathrm{Np}, \mathrm{Pu}$ \\
\hline $\mathrm{PaO}_{2} \cdot 2 \mathrm{Ta}_{2} \mathrm{O}_{5}[\mathrm{a}]$ & $\mathrm{PaO}_{2}+\mathrm{Ta}_{2} \mathrm{O}_{5} / 1250^{\circ} \mathrm{C} / 8 \mathrm{~h} /$ Vakuum & & 7,77 & & 7,79 & & $\mathrm{U} . \mathrm{Np}, \mathrm{Pu}$ \\
\hline $\mathrm{Pa}_{2} \mathrm{O}_{5} \cdot 3 \mathrm{Nb}_{2} \mathrm{O}_{5}$ & $\mathrm{~Pa}_{2} \mathrm{O}_{5}+\mathrm{Nb}_{2} \mathrm{O}_{5} / 1150^{\circ} \mathrm{C} / 8 \mathrm{~h} / \mathrm{O}_{2}$ & hexagonal, UTa $\mathrm{O}_{10,67}$ & 7,48 & & 15,81 & & $\mathrm{UNb}_{3} \mathrm{O}_{10,67}$ \\
\hline $\mathrm{Pa}_{2} \mathrm{O}_{5} \cdot 3 \mathrm{Ta}_{2} \mathrm{O}_{5}$ & $\mathrm{~Pa}_{2} \mathrm{O}_{5}+\mathrm{Ta}_{2} \mathrm{O}_{5} / 1200^{\circ} \mathrm{C} / 8 \mathrm{~h} / \mathrm{O}_{2}$ & & 7,425 & & 15,76 & & $\mathrm{NpNbO}_{9,5}$ \\
\hline
\end{tabular}

[a] Konnte nicht in reiner Form dargestellt werden, enthelt stets noch wechselnde Mengen $\mathrm{Pa}(\mathrm{V})$.

[b] S.E. = seltene Erdmetalle.

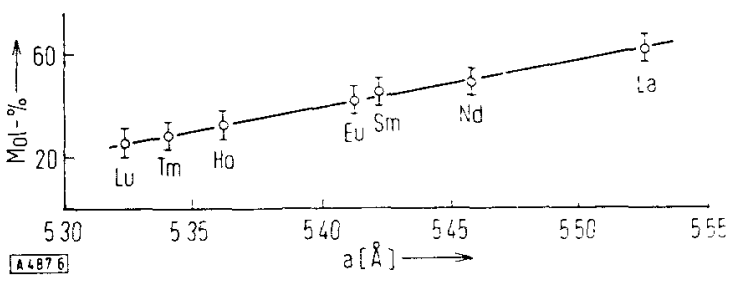

Abb. 6. Ioslichkeit von $\mathrm{MO}_{1,5}$ in $\left(\mathrm{M}_{0,5}^{\mathrm{III}} \mathrm{Pa}_{0,5} \mathrm{~V}_{2}\right) \mathrm{O}_{2}$.

Ordinate: Loslichkeit [Mol- \%]

Abszisse: Gitterkonstante $\mathfrak{a}[\AA]$ von $\left(\mathrm{M}_{0,5}^{111} \mathrm{~Pa}_{0},{ }_{5}\right) \mathrm{O}_{2}$

$\mathrm{Li}_{2} \mathrm{O} / \mathrm{U}_{2} \mathrm{O}_{5}$ eine Fluoritphase $[174,175]$. Einzig in Verbindungen des Typs $\mathrm{Ba}\left(\mathrm{M}_{0,5}^{\mathrm{II}}, \mathrm{Pa}_{0,5}\right) \mathrm{O}_{2,75}$ und $\mathrm{Ba}\left(\mathrm{M}_{0,5}^{\mathrm{III}}, \mathrm{Pa}_{0,5}\right) \mathrm{O}_{3}$ mit geordneter Fluoritstruktur ist eine Verwandtschaft des Protactiniums zu Niob und Tantal zu erkennen, allerdings konnte zumindest vom zuletzt

[165] C. Keller, Colloque International sur la Physico-Chimie du Protactinium, Orsay/Frankreich, Julı 1965.

[166] C. Keller, Kernforschungszentrum Karlsruhe, Bericht KFK-225 (1965).

[167] C. Keller, J. inorg. nuclear Chem. 26, 2069 (1964).

[168] C. Keller, J. inorg. nuclear Chem. 27, 1233 (1965).

[169] C. Keller, J. inorg. nuclear Chem. 27, 321 (1965).

[170] C. Keller u. K. H. Walter, J. norg. nuclear Chem. 27, 1253 (1965).

[171] C. Keller, L. Koch u. K. H. Walter, J. inorg. nuclear Chem. 27,1255 (1965).

[172] C. Keller, J. inorg. nuclear Chem. 27, 797 (1965).

[173] P. N. Iyer u. A. J. Smith, Colloque International sur la Physico-Chime du Protactinium, Orsay/Frankreich, Julı 1965. [174] W. Rütorff, $S$. Kemmler u. H. Leutner, Angew. Chem. 74, 429 (1962)

[175] L. M. Kovba u. A. N. Golubenko, J. Strukturchem. (russ.) $1,390(1960)$. genannten Typ auch die Uran-Verbindungen dargestellt werden [176].

\subsection{Nitride, Carbide und Sulfide}

Über die Systeme $\mathrm{Pa} / \mathrm{N}, \mathrm{Pa} / \mathrm{C}$ und $\mathrm{Pa} / \mathrm{S}$ liegen bisher nur sehr wenige Angaben vor ${ }^{[153]}$. Eine thermische Behandlung von $\mathrm{PaCl}_{5}$ mit $\mathrm{H}_{2} \mathrm{~S} / \mathrm{CS}_{2}$ bei $900^{\circ} \mathrm{C}$ fuhrt zur Bildung von PaOS, eine Behandlung von $\mathrm{PaCl}_{5}$ oder $\mathrm{PaCl}_{4}$ mit $\mathrm{NH}_{3}$-Gas bei $800^{\circ} \mathrm{C}$ zu $\mathrm{PaN}_{2}$. Die Identifizierung von $\mathrm{PaOS}$ und $\mathrm{PaN}_{2}$ erfolgte allein auf Grund der Isotypie mit den analogen Uranverbindungen. Durch Reaktion von $\mathrm{PaF}_{4}$ mit $\mathrm{Ba}+\mathrm{C}$ bei $1400^{\circ} \mathrm{C}$ laßt sich $\mathrm{PaC}$ darstellen.

\subsection{Nitratokomplexe des $\mathrm{Pa}(\mathrm{V})$}

Durch Reaktion von $\mathrm{N}_{2} \mathrm{O}_{5}$ mit $\mathrm{Pa}_{2} \mathrm{O}_{5} \cdot \mathrm{aq}$ oder $\mathrm{PaCl}_{5}$ erhält man die Hexanitratosäure $\mathrm{HPa}\left(\mathrm{NO}_{3}\right)_{6}{ }^{[157]}$, von der durch Reaktion der Hexahalogenoprotactinate( $(\mathrm{V})$ mit $\mathrm{N}_{2} \mathrm{O}_{5}$ auch Salze dargestellt werden konnen, z. B. $\mathrm{CsPa}\left(\mathrm{NO}_{3}\right)_{6}$ oder $\left[\mathrm{N}\left(\mathrm{CH}_{3}\right)_{4}\right] \quad \mathrm{Pa}\left(\mathrm{NO}_{3}\right)_{6}$. Dagegen bilden die Hexahalogenoniobate und -tantalate bei der analogen Reaktion nur die Oxotetranitratokomplexe, z. B. $\mathrm{CsNbO}\left(\mathrm{NO}_{3}\right)_{4}$. Alle diese Verbindungen sind gegen Hydrolyse sehr empfindlich, ihre Identifizierung erfolgte hauptsächlıch an Hand der IR-Spektren.

\section{Ausblick}

Unsere Kenntnisse von der Chemie des Protactiniums haben in den letzten Jahren so stark zugenommen, so daß wir heute einen umfassenden Überblick über die

[176] C. Keller u. B. Kanellakopulos, unveroffenthcht. 
Eigenschaften dieses Radioelements besitzen. Auf vielen Teilgebieten wissen wir über das Protactinium bereits viel besser Bescheid als über die homologen Elemente Niob und Tantal. Allerdings gibt es auf anderen Gebieten - wie Metallurgie, Phasendiagramme polynärer Systeme oder thermodynamische Daten - noch keine eingehenderen Untersuchungen, jedoch trifft dies auch noch für viele der ,bekannteren “Elemente zu. Wenn das

[177] P. J. Jones, Colloque International sur la Physico-Chimie du Protactinium, Orsay/Frankreich, Juli 1965. gègenwärtige Interes̉se am Protactinium weiter anhält und durch die Gewinnung einer größeren Menge an 231Pa noch gefördert werden könnte, dürften auch diese Lücken bald geschlossen werden, obwohl mit ${ }^{231} \mathrm{~Pa}$ in wägbaren Mengen infolge seiner Radiotoxizität (die maximal zulässige Konzentration von ${ }^{231} \mathrm{~Pa}$ in der Luft beträgt $10^{-8} \mathrm{mg} / \mathrm{m}^{3}$, für den als sehr giftig angesehenen Cyanwasserstoff jedoch $10 \mathrm{mg} / \mathrm{m}^{3}$ ) nur in dichten Manipulierkästen gearbeitet werden darf.

Eingegangen am 1. Oktober 1965

[A 487] 